\title{
Cutpoint Temperature Surrogate Modeling for Distillation Yields and Properties
}

Robert E. Franzoi ${ }^{a}$, Brenno C. Menezes ${ }^{b}$, Jeffrey D. Kellyc, Jorge A. W. Gut ${ }^{a,},{ }^{*}$, Ignacio E.

Grossmann $^{d}$

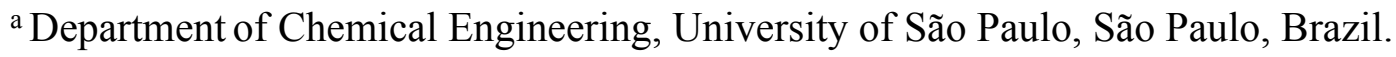

${ }^{\mathrm{b}}$ Division of Engineering Management and Decision Sciences, College of Science and

Engineering, Hamad Bin Khalifa University, Qatar Foundation, Doha, Qatar

c Industrial Algorithms Ltd., 15 St. Andrews Road, Toronto, Canada.

${ }^{\mathrm{d}}$ Chemical Engineering Department, Carnegie Mellon University, Pittsburgh, United States.

*Corresponding Author. E-mail address: jorgewgut@usp.br (J. A. W. Gut) 


\section{Supporting Information}

\section{Appendix A - Review on Distillation Unit Modeling}

\section{A1. Fixed yield modeling (FY)}

A straightforward method to model a crude distillation unit (CDU) is by using single or multiple fixed yield values for the CDU product stream outputs. This approach is largely used for strategic, tactical, and operational planning in industry, and consists in specifying the final-cuts or product distillates that divide the CDU fractions considering crude oil assay information to calculate these fractions. As this method uses perfect (sharp) fractionation to calculate the CDU yields, it does not rigorously compute the distillation column fractionation. When the fixed-yield formulation is addressed, the crude oil assay data and fixed boiling point temperature ranges without overlapping (sharp fractionation) are used to calculate the yields and properties for the final cuts. Hence, the distillates in the proposed problem were assigned to a certain range of micro-cuts or pseudocomponents, as shown in Table S1.

Table S1: Micro-cut range for the fixed yield model.

\begin{tabular}{cccc}
\hline Distillate & Initial micro-cut & Final micro-cut & $\begin{array}{c}\text { Number of } \\
\text { micro-cuts }\end{array}$ \\
\hline FG - Fuel gas & CH4 & C2H5 & 02 \\
LPG - Liquefied petroleum gas & C3H8 & NC4H10 & 03 \\
N - Naphtha & IC5H12 & CUT150 & 14 \\
K - Kerosene & CUT160 & CUT240 & 09 \\
LD - Light diesel & CUT250 & CUT320 & 08 \\
HD - Heavy diesel & CUT330 & CUT350 & 03 \\
ATR - Atmospheric residue & CUT360 & CUT850 & 50 \\
\hline
\end{tabular}

In Equation (S1), the inlet volumetric flows $Q_{c, c d u}$ for each crude oil $c$ incoming to the CDU are summed to calculate the overall volumetric feed flow $Q_{c d u}$ for the CDU. 


$$
Q_{c d u}=\sum_{c} Q_{c, c d u}
$$

The volumetric flow rate $Q_{c u t}^{f c}$ for each distillate or final cut $f c$ can be calculated as the summation of yields of micro-cuts $Y L D_{m c}$ that flow to the pre-defined boiling point temperature ranges, resulting in the product of the overall volumetric flow in the CDU and the summation of predefined micro-cuts yields to each cut $f c$ in Equation (S2). The set $M C_{f c}$ can be determined from Table A1 and represents the micro cuts related to final cuts of distillates.

$$
Q_{c u t}^{f c}=Q_{c d u} \sum_{m c} Y L C_{f c} D_{m c} \quad \forall c u t \in F C
$$

Volume- and mass-based mixing rules are used to calculate the properties of each final cut $f c$. Equation (S3) represents the volumetric rules for specific gravity, and Equation (S4) represents the mass rules for sulfur content. Thus, yields and properties (volume- and mass-based) for the final cuts can be calculated from the crude-oil assay data using Equations (S3) and (S4).

$$
\begin{aligned}
& V P_{c u t}^{f c}=\frac{\sum_{m c \in M C_{f c}} V P_{m c}^{f c} Q_{m c}}{\sum_{m c \in M C_{f c}} Q_{m c}} \quad \forall f c \\
& M P_{c u t}^{f c}=\frac{\sum_{m c \in M C_{f c}} M P_{m c}^{f c} V P_{m c}^{f c} Q_{m c}}{\sum_{m c \in M C_{f c}} V P_{m c}^{f c} Q_{m c}} \quad \forall f c
\end{aligned}
$$

In order to improve the prediction of the crude oil distillation unit outputs, there are models that consider non-sharp fractionation by including volume and/or mass variations for the CDU products of external distillates by creating hypothetical internal streams that can flow to the respective upper and lower final distillates, the so-called light and heavy swing-cuts, as shown in the swing-cut modeling as follows.

\section{A2. Conventional swing-cut modeling (CSW)}

The swing-cut method creates additional degrees of freedom by allowing the optimization of each swing-cut amount flowing to the lighter and heavier final distillates. ${ }^{18}$ The swing-cuts are hypothetical internal modeling constructs, and do not physically exist in the tower. In this work, 
three swing-cuts $S C$ are considered. Table S2 shows the initial and final micro cuts, and the respective number of micro-cuts related to each distillate.

Table S2: Micro-cuts for the swing-cut model.

\begin{tabular}{cccc}
\hline Distillate & Initial Micro-cut & Final Micro-cut & $\begin{array}{c}\text { Number of } \\
\text { Micro-cuts }\end{array}$ \\
\hline FG - Fuel gas & CH4 & C2H5 & 02 \\
LPG - Liquefied petroleum gas & C3H8 & NC4H10 & 03 \\
N - Naphtha & IC5H12 & CUT120 & 11 \\
SW1 - Swing-cut 1 & CUT130 & CUT150 & 03 \\
K - Kerosene & CUT160 & CUT200 & 05 \\
SW2 - Swing-cut 2 & CUT210 & CUT240 & 04 \\
LD - Light diesel & CUT250 & CUT290 & 05 \\
SW3 - Swing-cut 3 & CUT300 & CUT320 & 03 \\
HD - Heavy diesel & CUT330 & CUT360 & 04 \\
ATR - Atmospheric residue & CUT370 & CUT850 & 49 \\
\hline
\end{tabular}

The mathematical model for the conventional swing-cut method is given as follows. Equations (S1) to (S4) are the same as in the fixed yield model, which represent a mass balance for the CDU, the flow calculation for each distillate, and the volume- and mass-based balances, respectively. However, some of the final cuts in the fixed yield model are intermediate cuts in the swing-cut model, which will be further blended with the swing-cuts to create the final cuts. Thus, Equation (S5) represents the mass balances for the intermediate cuts (naphtha, kerosene, light diesel, heavy diesel, and the three swing-cuts), with $Q_{c u t}^{i c}$ as their flows.

$$
Q_{c u t}^{i c}=Q_{c d u} \sum_{m c} Y L D_{m c} \quad \forall c u t \in I C
$$

By the definition of swing-cut methods, Equation (S6) represents the mass balance when the swing-cut splits to its light and heavy fractions, which are the additional decision variables included in the optimization problem.

$$
Q_{s w}=Q_{s w}^{\text {light }}+Q_{s w}^{\text {heavy }} \quad \forall s w \in S W
$$


When intermediate cuts are mixed with their respective swing-cuts, we use material and property balance constraints to calculate the flows and properties for the final cuts. Equations (S7), (S8) and (S9) calculate the flows, the volume-based properties, and the mass-based properties, respectively, for the final cuts of each distillate related to swing-cuts.

$$
\begin{aligned}
& Q_{N}^{f c}=Q_{N}^{i c}+Q_{s w 1}^{l i g h t} \\
& Q_{K}^{f c}=Q_{K}^{i c}+Q_{s w 1}^{h e a v y}+Q_{s w 2}^{l i g h t} \\
& Q_{L D}^{f c}=Q_{L D}^{i c}+Q_{s w 2}^{h e a v y}+Q_{s w 3}^{\text {light }} \\
& Q_{H D}^{f c}=Q_{H D}^{i c}+Q_{s w 3}^{h e a v y} \\
& V P_{N}^{f_{c}}=\frac{Q_{N}^{i c} V P_{N}^{i c}+Q_{s w 1}^{l i g h t} V P_{s w 1}}{Q_{N}^{i c}+Q_{s w 1}^{l i g h t}} \\
& V P_{K}^{f c}=\frac{Q_{K}^{i c} V P_{K}^{i c}+Q_{s w 1}^{\text {heavy } V} P_{s w 1}+Q_{s w 2}^{\text {light }} V P_{s w 2}}{Q_{K}^{i c}+Q_{s w 1}^{\text {heavy }}+Q_{s w 2}^{\text {light }}} \\
& V P_{L D}^{f c}=\frac{Q_{L D}^{i c} V P_{L D}^{i c}+Q_{s w 2}^{\text {heavy }} V P_{s w 2}+Q_{s w 3}^{\text {light }} V P_{s w 3}}{Q_{L D}^{i c}+Q_{s w 2}^{\text {heavy }}+Q_{s w 3}^{\text {light }}} \\
& V P_{H D}^{f c}=\frac{Q_{H D}^{i c} V P_{H D}^{i c}+Q_{s w 3}^{h e a v y} V P_{s w 3}}{Q_{H D}^{i c}+Q_{s w 3}^{\text {heavy }}} \\
& M P_{N}^{f c}=\frac{Q_{N}^{i c} V P_{N}^{i c} M P_{N}^{i c}+Q_{s w 1}^{l i g h t} V P_{s w 1} M P_{s w 1}}{Q_{N}^{i c} V P_{N}^{i c}+Q_{s w 1}^{l i g h t} V P_{s w 1}} \\
& M P_{K}^{f c}=\frac{Q_{K}^{i c} V P_{K}^{i c} M P_{K}^{i c}+Q_{s w 1}^{h e a v y} V P_{s w 1} M P_{s w 1}+Q_{s w 2}^{l i g h t} V P_{s w 2} M P_{s w 2}}{Q_{K}^{i c} V P_{K}^{i c}+Q_{s w 1}^{h e a v y} V P_{s w 1}+Q_{s w 2}^{l i g h t} V P_{s w 2}} \\
& M P_{L D}^{f c}=\frac{Q_{N}^{i c} V P_{N}^{i c} M P_{N}^{i c}+Q_{s w 2}^{h e a v y} V P_{s w 2} M P_{s w 2}+Q_{s w 3}^{l i g h t} V P_{s w 3} M P_{s w 3}}{Q_{N}^{i c} V P_{N}^{i c}+Q_{s w 2}^{h e a v y} V P_{s w 2}+Q_{s w 3}^{l i g h t} V P_{s w 3}} \\
& M P_{H D}^{f c}=\frac{Q_{N}^{i c} V P_{N}^{i c} M P_{N}^{i c}+Q_{s w 3}^{h e a v y} V P_{s w 3} M P_{s w 3}}{Q_{N}^{i c} V P_{N}^{i c}+Q_{s w 3}^{h e a v y} V P_{s w 3}}
\end{aligned}
$$


The conventional swing-cut method creates additional degrees of freedom by allowing the optimization of each swing-cut amount flowing to the lighter and heavier final distillates. Therefore, the search space for optimization becomes larger and an equivalent or better solution is expected when compared to the (multiple) fixed yield method.

\section{A3. Improved swing-cut modeling (ISW)}

The conventional swing-cut modeling considers fixed properties for the hypothetical cuts that swing between adjacent light and heavy distillates, although that may lead to inaccuracies in the quantity and quality predictions of the final distillates. ${ }^{5}$ In the improved swing-cut modeling, each swing-cut is split into two internal light and heavy streams, similarly to the CSW modeling. However, the light and heavy streams are not assumed to have the bulk quality. The ISW method proposed by these authors adds a set of interpolations to improve the prediction of distillates by considering quality variations for the light and heavy fractions of each swing-cut. If the whole swing-cut flows to a specific fraction (either light or heavy), the properties of this fraction will be the bulk properties of the swing-cut. However, whenever the swing-cut splits to both fractions, there is a difference among their properties, which are also different from the bulk. Any light swing-cut stream that flows to the upper cut will have a lighter property than the swing-cut bulk (lower value in the vast majority of the properties, such as density and sulphur concentration), and heavier than the interface between the bulk and the upper cut. Similarly, a heavy swing-cut stream that flows to the lower cut will have a heavier property than the bulk swing-cut (typically higher value when compared with the bulk) and lighter than the interface between the bulk and the lower cut.

The mathematical model for the improved swing-cut method and a brief explanation about how this method mathematically works is given as follows. A complete explanation and additional details can be found in Menezes et al. ${ }^{5}$ The model for the improved swing-cut uses Equations (S1) to (S9) from the conventional swing-cut method. The main contribution to the improved swingcut method regards the swing-cut properties calculation. Each swing-cut may be split into two internal streams with different qualities, whereas the conventional swing-cut uses the bulk quality for both streams. Interpolations are performed to better predict the qualities of final distillates, which vary linearly between the properties at their adjacent hypothetical interfaces. Besides, new 
variables are created to represent these interfaces between adjacent cuts, VPI for volume-based properties and MPI for mass-based properties, and their calculation is performed considering the blending of micro-cut streams, such as Equations (S10) and (S11), in which $m c 1$ and $m c 2$ are the lighter and heavier adjacent micro cuts to the respective hypothetical interface of the swing-cut.

$$
\begin{array}{ll}
V P I_{s W}=\frac{V P_{m c 1} Q_{m c 1}+V P_{m c 2} Q_{m c 2}}{Q_{m c 1}+Q_{m c 2}} & \forall s w \in S W \\
M P I_{s W}=\frac{V P_{m c 1} M P_{m c 1} Q_{m c 1}+V P_{m c 2} M P_{m c 2} Q_{m c 2}}{V P_{m c 1} Q_{m c 1}+V P_{m c 2} Q_{m c 2}} & \forall s w \in S W
\end{array}
$$

Equations (S12) and (S13) use the interface variables and calculate the light and heavy swing-cut volume-based properties using linear interpolation around the bulk and hypothetical light and heavy interfaces of the swing-cuts.

$$
\begin{array}{cc}
V P_{s W}^{\text {light }}=V P I_{s W}^{\text {light }}+\frac{V P_{s w}-V P I_{s w}^{\text {light }}}{Q_{s w}} Q_{s w}^{\text {light }} & \forall s w \in S W \\
V P_{s w}^{\text {heavy }}=V P I_{s w}^{\text {heavy }}+\frac{V P_{s w}-V P I_{s w}^{\text {heavy }}}{Q_{s w}} Q_{s w}^{\text {heavy }} & \forall s w \in S W
\end{array}
$$

Similarly, Equations (S14) and (S15) applies linear interpolation to calculate mass-based properties.

$$
\begin{array}{ll}
M P_{s w}^{\text {light }}=M P I_{s w}^{\text {light }}+\frac{M P_{s w}-M P I_{s w}^{\text {light }}}{V P_{s w} Q_{s w}} V P_{s w}^{\text {light }} Q_{s w}^{\text {light }} & \forall s w \in S W \\
M P_{s w}^{\text {heavy }}=M P I_{s w}^{\text {heavy }}+\frac{M P_{s w}-M P I_{s w}^{\text {heavy }}}{V P_{s w} Q_{s w}} V P_{s w}^{\text {heavy }} Q_{s w}^{\text {heavy }} & \forall s w \in S W
\end{array}
$$

These quality interpolation constraints are used for improvements in the predictions of the final distillates properties and provide a more accurate modeling for the swing-cut method. 


\section{Appendix B - Data Set 1: Independent and Dependent Variables}

Table S3 shows data set 1 used to test the proposed methodology, generated by the Latin Hypercube Sampling technique. There are seven independent variables; $\mathrm{x}_{1}$ to $\mathrm{x}_{4}$ account for the four crude oils whereas $\mathrm{x}_{5}$ to $\mathrm{x}_{7}$ account for the three swing-cuts.

Table S3: Independent data set 1 generated by Latin Hypercube Sampling

\begin{tabular}{|c|c|c|c|c|c|c|}
\hline $\mathrm{x} 1$ & $\mathrm{x} 2$ & $\mathrm{x} 3$ & $\mathrm{x} 4$ & $\mathrm{x} 5$ & $\mathrm{x} 6$ & $\mathrm{x} 7$ \\
\hline 0.115977805 & 0.033017147 & 0.398073556 & 0.452931491 & 0.150655345 & 0.244379184 & 0.250940892 \\
\hline 0.17063455 & 0.304731891 & 0.356932792 & 0.167700766 & 0.680388327 & 0.626619307 & 0.690691442 \\
\hline 0.437511765 & 0.000262226 & 0.345845439 & 0.21638057 & 0.877668313 & 0.891336845 & 0.948349719 \\
\hline 0.06653081 & 0.297344795 & 0.275739844 & 0.360384551 & 0.410538576 & 0.111839298 & 0.983078379 \\
\hline 0.152055211 & 0.399795334 & 0.291271843 & 0.156877611 & 0.01524906 & 0.15094929 & 0.094721638 \\
\hline 0.393300459 & 0.333894058 & 0.144234342 & 0.128571141 & 0.607306773 & 0.674940778 & 0.659651006 \\
\hline 0.010596159 & 0.165004684 & 0.442102947 & 0.38229621 & 0.712632694 & 0.327694143 & 0.735454199 \\
\hline 0.038434607 & 0.38827486 & 0.190178029 & 0.383112504 & 0.595498137 & 0.6371853 & 0.323330144 \\
\hline 0.217176484 & 0.280328505 & 0.314511862 & 0.187983149 & 0.568093062 & 0.490090458 & 0.810325839 \\
\hline 0.109132126 & 0.104175936 & 0.499419653 & 0.287272285 & 0.391814658 & 0.818955285 & 0.791474982 \\
\hline 0.08831644 & 0.417232906 & 0.16525176 & 0.329198894 & 0.160594948 & 0.279288916 & 0.728819599 \\
\hline 0.059161441 & 0.555996951 & 0.192046255 & 0.192795352 & 0.242507308 & 0.380317354 & 0.203769106 \\
\hline 0.631079851 & 0.241088534 & 0.114511387 & 0.013320228 & 0.999989158 & 0.217771444 & 0.924663898 \\
\hline 0.047267418 & 0.252543914 & 0.383151728 & 0.31703694 & 0.086919631 & 0.978241615 & 0.596830213 \\
\hline 0.301732708 & 0.041790985 & 0.422001624 & 0.234474683 & 0.355744247 & 0.053566554 & 0.6430744 \\
\hline 0.162560335 & 0.356109954 & 0.083705345 & 0.397624366 & 0.429969602 & 0.859107215 & 0.934965853 \\
\hline 0.556992807 & 0.332220876 & 0.057359512 & 0.053426805 & 0.02319535 & 0.12425503 & 0.994294199 \\
\hline 0.574136288 & 0.095049313 & 0.148851646 & 0.181962754 & 0.628285865 & 0.340537526 & 0.164196392 \\
\hline 0.092649922 & 0.312104146 & 0.381446414 & 0.213799518 & 0.537739813 & 0.953041174 & 0.573006701 \\
\hline 0.236260597 & 0.524800342 & 0.108995724 & 0.129943337 & 0.89295066 & 0.781743732 & 0.756911513 \\
\hline 0.427723623 & 0.153049757 & 0.187589264 & 0.231637356 & 0.259666102 & 0.478175334 & 0.672836395 \\
\hline 0.31169184 & 0.26910479 & 0.212191229 & 0.207012141 & 0.889221903 & 0.732520616 & 0.523995144 \\
\hline 0.117632609 & 0.38795601 & 0.053727691 & 0.44068369 & 0.382432681 & 0.59606146 & 0.374956835 \\
\hline 0.322007089 & 0.258974325 & 0.307061522 & 0.111957064 & 0.64803347 & 0.568529948 & 0.292840421 \\
\hline 0.273043214 & 0.220208361 & 0.473145391 & 0.033603034 & 0.971843473 & 0.373243971 & 0.621421271 \\
\hline 0.237883639 & 0.215175385 & 0.209803636 & 0.33713734 & 0.049106146 & 0.586990483 & 0.559046003 \\
\hline 0.340726865 & 0.345626102 & 0.249014753 & 0.06463228 & 0.233591847 & 0.228178438 & 0.225002512 \\
\hline 0.477200204 & 0.202741417 & 0.246580943 & 0.073477436 & 0.58635123 & 0.105116871 & 0.2392449 \\
\hline 0.204828615 & 0.156521522 & 0.196053062 & 0.442596801 & 0.203779618 & 0.924044133 & 0.63974291 \\
\hline 0.30480881 & 0.127817029 & 0.365191246 & 0.202182916 & 0.830288693 & 0.872068679 & 0.44828057 \\
\hline 0.225846078 & 0.13774912 & 0.289218145 & 0.347186657 & 0.077385275 & 0.904312847 & 0.036014407 \\
\hline 0.28250372 & 0.339768573 & 0.114112391 & 0.263615315 & 0.900499748 & 0.179266455 & 0.101303877 \\
\hline 0.002539167 & 0.410328186 & 0.52547982 & 0.061652826 & 0.673991095 & 0.338332678 & 0.397317653 \\
\hline 0.557116869 & 0.402118027 & 0.002191236 & 0.038573868 & 0.48978725 & 0.83431064 & 0.218920469 \\
\hline 0.281609622 & 0.399572796 & 0.049989996 & 0.268827586 & 0.449350636 & 0.096132998 & 0.487303161 \\
\hline 0.196046454 & 0.433687785 & 0.134227674 & 0.236038088 & 0.812327007 & 0.160011901 & 0.38001758 \\
\hline 0.316425842 & 0.33662573 & 0.050049906 & 0.296898521 & 0.213649885 & 0.50361124 & 0.114105238 \\
\hline 0.319104695 & 0.15994204 & 0.046221621 & 0.474731643 & 0.611634752 & 0.195280097 & 0.462591437 \\
\hline 0.132824617 & 0.230353351 & 0.618761274 & 0.018060758 & 0.529043193 & 0.538950901 & 0.047797072 \\
\hline 0.071724645 & 0.095630889 & 0.710389988 & 0.122254478 & 0.745677304 & 0.578449196 & 0.605643118 \\
\hline 0.299835468 & 0.253401121 & 0.189506118 & 0.257257293 & 0.634881085 & 0.396394522 & 0.342729099 \\
\hline 0.308821689 & 0.187756368 & 0.167864923 & 0.33555702 & 0.433154624 & 0.71975874 & 0.085143373 \\
\hline 0.0362072 & 0.406903808 & 0.149448948 & 0.407440044 & 0.703920146 & 0.455895396 & 0.853924856 \\
\hline 0.413609157 & 0.308696295 & 0.261500574 & 0.016193975 & 0.131964333 & 0.984540154 & 0.806375558 \\
\hline 0.261570354 & 0.536580972 & 0.135339552 & 0.066509123 & 0.361078227 & 0.141759778 & 0.076593328 \\
\hline 0.075749103 & 0.276913337 & 0.375837866 & 0.271499694 & 0.177204514 & 0.606265314 & 0.711136716 \\
\hline 0.217669165 & 0.253620523 & 0.291287758 & 0.237422554 & 0.296214777 & 0.521762382 & 0.280361672 \\
\hline
\end{tabular}




\begin{tabular}{|c|c|c|c|c|c|c|}
\hline 0.240834669 & 0.594453974 & 0.018403926 & 0.146307431 & 0.108937319 & 0.869517924 & 0.897756624 \\
\hline 0.320045522 & 0.189543543 & 0.161998148 & 0.328412787 & 0.003435683 & 0.423524738 & 0.560278727 \\
\hline 0.225603917 & 0.14154767 & 0.300618144 & 0.332230269 & 0.828803262 & 0.946431202 & 0.549208683 \\
\hline 0.230464911 & 0.190838634 & 0.140540782 & 0.438155673 & 0.519730053 & 0.073007878 & 0.173401968 \\
\hline 0.27597789 & 0.268306593 & 0.173932071 & 0.281783447 & 0.910299011 & 0.265484195 & 0.242859578 \\
\hline 0.261702128 & 0.116741071 & 0.293340567 & 0.328216234 & 0.794934663 & 0.236879507 & 0.123880473 \\
\hline 0.130062949 & 0.254380426 & 0.464180228 & 0.151376397 & 0.194138999 & 0.434156526 & 0.268732769 \\
\hline 0.263131768 & 0.015260785 & 0.134008205 & 0.587599241 & 0.755590235 & 0.025077255 & 0.773423579 \\
\hline 0.309993093 & 0.306674423 & 0.098405294 & 0.28492719 & 0.848066024 & 0.01565984 & 0.584933583 \\
\hline 0.075578937 & 0.159223085 & 0.287535682 & 0.477662296 & 0.181336995 & 0.290880454 & 0.667794628 \\
\hline 0.267825028 & 0.396272588 & 0.24328753 & 0.092614854 & 0.284186397 & 0.650773226 & 0.865605778 \\
\hline 0.102212235 & 0.275506549 & 0.415182877 & 0.207098339 & 0.86018695 & 0.062075946 & 0.270419201 \\
\hline 0.29143001 & 0.368151307 & 0.096860351 & 0.243558332 & 0.953205478 & 0.464475275 & 0.065940442 \\
\hline 0.463527356 & 0.088446431 & 0.181834004 & 0.266192209 & 0.348929792 & 0.933006231 & 0.685718848 \\
\hline 0.152557545 & 0.069329974 & 0.429506921 & 0.34860556 & 0.056623499 & 0.201149505 & 0.02973766 \\
\hline 0.385629502 & 0.441622049 & 0.008044685 & 0.164703764 & 0.577551008 & 0.139793034 & 0.361523459 \\
\hline 0.240648997 & 0.20826862 & 0.164422086 & 0.386660297 & 0.452450138 & 0.619467113 & 0.433765516 \\
\hline 0.250463778 & 0.333763157 & 0.149350382 & 0.266422682 & 0.461057243 & 0.04908057 & 0.9071385 \\
\hline 0.367264685 & 0.131106095 & 0.045361441 & 0.45626778 & 0.038486717 & 0.88626096 & 0.147958285 \\
\hline 0.233267688 & 0.202880328 & 0.272083179 & 0.291768806 & 0.125272405 & 0.963310069 & 0.912331421 \\
\hline 0.344916173 & 0.237902907 & 0.314987275 & 0.102193645 & 0.473519485 & 0.761987186 & 0.318638166 \\
\hline 0.269318229 & 0.038744906 & 0.408123023 & 0.283813841 & 0.772472747 & 0.809466924 & 0.130592706 \\
\hline 0.120934507 & 0.208724113 & 0.343478556 & 0.326862824 & 0.305003105 & 0.407193157 & 0.615382281 \\
\hline 0.217846446 & 0.257689014 & 0.310000455 & 0.214464085 & 0.505135404 & 0.030727352 & 0.764611438 \\
\hline 0.270065621 & 0.409460865 & 0.18311659 & 0.137356924 & 0.337429315 & 0.824496024 & 0.47467383 \\
\hline 0.01162043 & 0.349374611 & 0.345123337 & 0.293881622 & 0.320952772 & 0.08323987 & 0.822498898 \\
\hline 0.407398326 & 0.009558082 & 0.360509075 & 0.222534517 & 0.69035889 & 0.661870913 & 0.704437433 \\
\hline 0.145080225 & 0.307262722 & 0.442527866 & 0.105129188 & 0.805438883 & 0.701307125 & 0.158855807 \\
\hline 0.265121486 & 0.283910615 & 0.044177925 & 0.406789975 & 0.724865479 & 0.484106826 & 0.003427587 \\
\hline 0.202870721 & 0.121065449 & 0.317291614 & 0.358772215 & 0.261406704 & 0.442476975 & 0.300526757 \\
\hline 0.293687025 & 0.284408811 & 0.374459719 & 0.047444445 & 0.657371243 & 0.51847781 & 0.356544551 \\
\hline 0.219150784 & 0.15235821 & 0.026964449 & 0.601526558 & 0.406731957 & 0.183995822 & 0.197781205 \\
\hline 0.303318171 & 0.254919324 & 0.213692313 & 0.228070192 & 0.769101448 & 0.998033844 & 0.83480881 \\
\hline 0.268282681 & 0.309261801 & 0.41827218 & 0.004183338 & 0.942099383 & 0.644327723 & 0.506040373 \\
\hline 0.389320368 & 0.193811382 & 0.061390148 & 0.355478103 & 0.924231289 & 0.745273259 & 0.51765643 \\
\hline 0.210047903 & 0.23487911 & 0.347124409 & 0.207948579 & 0.859826862 & 0.690064576 & 0.455322383 \\
\hline 0.041159738 & 0.593529257 & 0.157349342 & 0.207961663 & 0.542680783 & 0.285917113 & 0.878920891 \\
\hline 0.328196369 & 0.074580152 & 0.350210192 & 0.247013286 & 0.73584936 & 0.550193165 & 0.53651673 \\
\hline 0.343546437 & 0.236163906 & 0.098295602 & 0.321994056 & 0.372635265 & 0.250906814 & 0.180828409 \\
\hline 0.32936563 & 0.233719957 & 0.205107654 & 0.231806759 & 0.938582996 & 0.364410311 & 0.784091258 \\
\hline 0.200587377 & 0.298082185 & 0.233605482 & 0.267724956 & 0.31523557 & 0.724231425 & 0.977567324 \\
\hline 0.154625684 & 0.046087334 & 0.287853094 & 0.511433888 & 0.147223749 & 0.689544766 & 0.428890384 \\
\hline 0.137319112 & 0.453727219 & 0.326253934 & 0.082699735 & 0.091831764 & 0.006457821 & 0.966591577 \\
\hline 0.025812305 & 0.075056895 & 0.744091365 & 0.155039435 & 0.069746495 & 0.549338193 & 0.847010612 \\
\hline 0.197615367 & 0.498781179 & 0.261374731 & 0.042228723 & 0.662790466 & 0.779361495 & 0.408638294 \\
\hline 0.201585499 & 0.307412926 & 0.090767574 & 0.400234001 & 0.223184322 & 0.318905005 & 0.746426674 \\
\hline 0.284005445 & 0.094593526 & 0.341205532 & 0.280195497 & 0.961930765 & 0.350375465 & 0.490448101 \\
\hline 0.360018284 & 0.134038875 & 0.42028751 & 0.085655331 & 0.274743498 & 0.753969207 & 0.410455991 \\
\hline 0.314523455 & 0.032074291 & 0.286350436 & 0.367051817 & 0.78281421 & 0.30842235 & 0.014433129 \\
\hline 0.487925509 & 0.077371378 & 0.281812535 & 0.152890578 & 0.553822529 & 0.845246878 & 0.334273906 \\
\hline 0.258761573 & 0.145742821 & 0.357453076 & 0.238042531 & 0.49860892 & 0.410119392 & 0.05662512 \\
\hline 0.283075476 & 0.111305208 & 0.089537145 & 0.516082172 & 0.98981634 & 0.913228388 & 0.959522311 \\
\hline 0.218288194 & 0.20228937 & 0.368913784 & 0.210508652 & 0.113340986 & 0.791948651 & 0.880974726 \\
\hline
\end{tabular}


Table S4 shows the dependent variables for yields calculated using the Improved Swing-Cut Method from data set 1 .

Table S4: Dependent variables for yields of distillates from data set 1

\begin{tabular}{|c|c|c|c|c|c|}
\hline$Y_{\mathrm{LPG}}$ & $Y_{N}$ & $\mathrm{Y}_{\mathrm{K}}$ & $\mathrm{Y}_{\mathrm{LD}}$ & $\mathrm{Y}_{\mathrm{HD}}$ & $\mathrm{Y}_{\mathrm{ATR}}$ \\
\hline 1.112979447 & 6.391693324 & 8.602618808 & 12.28891701 & 8.495072666 & 63.01181936 \\
\hline 1.072063092 & 8.52572334 & 10.15903877 & 12.87892558 & 6.260683754 & 60.99258915 \\
\hline 1.054074163 & 9.183486314 & 10.66630271 & 12.32009492 & 5.095686541 & 61.57402297 \\
\hline 1.07623991 & 7.196822774 & 7.728944012 & 16.99127767 & 4.969574266 & 61.9312527 \\
\hline 1.055327277 & 6.524707024 & 9.665623045 & 12.89293887 & 9.061538651 & 60.68594921 \\
\hline 0.996734221 & 8.551955198 & 11.08659676 & 12.56866125 & 6.404093078 & 60.27420124 \\
\hline 1.125690764 & 7.938020818 & 7.745318478 & 14.37779963 & 6.15301811 & 62.56038903 \\
\hline 1.058098703 & 7.61963125 & 10.17338087 & 11.14224673 & 8.120014715 & 61.77857741 \\
\hline 1.059461984 & 8.172713296 & 9.730837796 & 14.1709648 & 5.711972845 & 61.04304586 \\
\hline 1.124670153 & 7.397891996 & 11.30042447 & 12.0251454 & 5.846658475 & 62.20401675 \\
\hline 1.042996947 & 6.534520036 & 9.640285212 & 15.12597619 & 6.161733789 & 61.38359995 \\
\hline 1.039589608 & 7.034375682 & 10.39031521 & 12.33598285 & 8.573609003 & 60.50952396 \\
\hline 0.960971704 & 10.34100461 & 7.453845096 & 16.3635732 & 5.112323854 & 59.64662111 \\
\hline 1.101652578 & 6.418704612 & 13.20576879 & 10.43029734 & 6.775577193 & 61.96357198 \\
\hline 1.08553106 & 7.535092684 & 7.645737283 & 15.30044854 & 6.529798652 & 61.79909665 \\
\hline 1.022593064 & 7.192380609 & 11.96102912 & 12.83153224 & 5.217778601 & 61.66495477 \\
\hline 0.955199589 & 6.895456193 & 10.20221321 & 17.37354873 & 4.795714958 & 59.65534694 \\
\hline 0.989578574 & 8.623437466 & 8.888704505 & 11.75043539 & 8.787969081 & 60.846625 \\
\hline 1.088400601 & 7.937137017 & 12.21053192 & 10.51870197 & 6.833535099 & 61.30288683 \\
\hline 0.99919678 & 9.335847523 & 10.97405095 & 12.63607316 & 5.933095033 & 60.00121078 \\
\hline 1.014967721 & 7.276365559 & 10.59868669 & 13.44456443 & 6.400065269 & 61.15421383 \\
\hline 1.027624121 & 9.155450587 & 10.19009454 & 11.49072715 & 7.081466414 & 60.94199736 \\
\hline 1.022032553 & 6.92028075 & 10.5904758 & 11.62347288 & 7.921359896 & 61.81315452 \\
\hline 1.042852655 & 8.683317152 & 10.12380696 & 11.3131919 & 8.110306858 & 60.61282375 \\
\hline 1.08251272 & 9.852127911 & 8.000234557 & 13.87045511 & 6.517449485 & 60.56505481 \\
\hline 1.044363394 & 6.32872795 & 11.45129764 & 12.34207674 & 6.983817804 & 61.74226927 \\
\hline 1.022013327 & 7.492017266 & 9.762434831 & 13.07786072 & 8.40600499 & 60.12198138 \\
\hline 1.012183628 & 8.698254874 & 7.889744298 & 13.61061238 & 8.352844519 & 60.32041348 \\
\hline 1.052889202 & 6.533033405 & 12.42578254 & 10.84751199 & 6.656259231 & 62.38115796 \\
\hline 1.067541396 & 8.979218688 & 10.82166926 & 10.19718137 & 7.428687763 & 61.39799736 \\
\hline 1.066856113 & 6.421055817 & 12.8195656 & 8.068005148 & 9.464642001 & 62.05548482 \\
\hline 1.009412928 & 9.012821496 & 7.110468593 & 12.65054505 & 9.124621232 & 60.97846203 \\
\hline 1.118558003 & 8.662090572 & 8.661621862 & 13.25242387 & 7.556871853 & 60.63655793 \\
\hline 0.939455482 & 8.483847362 & 12.92793867 & 9.695169841 & 8.453207453 & 59.37528662 \\
\hline 0.99325345 & 7.62379369 & 8.095118599 & 15.07204295 & 7.295121014 & 60.80504191 \\
\hline 1.018460729 & 8.754408764 & 7.329153478 & 14.24308449 & 7.776520241 & 60.7632162 \\
\hline 0.993293508 & 6.873214154 & 10.99597719 & 10.89621722 & 9.09349848 & 61.03383632 \\
\hline 1.00842928 & 7.634543497 & 7.609387652 & 13.87092432 & 7.534421349 & 62.23648023 \\
\hline 1.129300535 & 8.450302457 & 10.10635619 & 10.26954345 & 9.153735301 & 60.78140154 \\
\hline 1.167217653 & 8.770823394 & 9.215253899 & 12.4005736 & 6.620636444 & 61.72346971 \\
\hline 1.02719793 & 8.247600964 & 8.999080984 & 12.4476089 & 7.969082747 & 61.19822196 \\
\hline 1.028091343 & 7.471550689 & 11.12386276 & 9.354329016 & 9.242577734 & 61.67136871 \\
\hline 1.04988613 & 7.854940948 & 8.880014559 & 14.69499899 & 5.601912634 & 61.80990984 \\
\hline 1.01547338 & 7.31726776 & 14.51751985 & 11.46546974 & 5.655224767 & 59.91029087 \\
\hline 0.998378404 & 7.802788981 & 9.066539629 & 13.21559778 & 9.113041663 & 59.68134677 \\
\hline 1.093477271 & 6.772108753 & 11.14467114 & 13.0100217 & 6.213303915 & 61.65992154 \\
\hline 1.057915816 & 7.254202798 & 10.58626509 & 11.44715604 & 8.239718295 & 61.30524393 \\
\hline 0.976765134 & 6.765418868 & 14.1596824 & 12.87943257 & 5.27208502 & 59.82379968 \\
\hline 1.025083982 & 6.24626421 & 10.81033635 & 13.21380644 & 6.980222913 & 61.61565037 \\
\hline 1.068470165 & 8.554457461 & 10.97686061 & 10.28116189 & 7.024098738 & 61.99025382 \\
\hline 1.036140673 & 7.413979839 & 7.190032242 & 13.17154688 & 8.884843037 & 62.19825311 \\
\hline 1.027142583 & 8.979312144 & 7.434455899 & 12.70159097 & 8.457292774 & 61.28935773 \\
\hline 1.063558629 & 8.498998067 & 7.329992068 & 11.98686144 & 9.037918264 & 61.97791313 \\
\hline
\end{tabular}




\begin{tabular}{|c|c|c|c|c|c|}
\hline 1.101476902 & 7.129759189 & 10.38181537 & 11.86944255 & 8.223241191 & 61.18577734 \\
\hline 1.045766035 & 7.69237437 & 5.959747772 & 15.87394637 & 6.099314121 & 63.23069724 \\
\hline 1.005262382 & 8.817260963 & 6.32697735 & 15.79577489 & 6.839592259 & 61.10220017 \\
\hline 1.089418159 & 6.347488091 & 8.908143119 & 14.2452995 & 6.531084284 & 62.77861753 \\
\hline 1.028436956 & 7.539640271 & 11.96019817 & 13.73337341 & 5.404873619 & 60.21613773 \\
\hline 1.095872271 & 8.890606902 & 6.361174021 & 13.91623109 & 8.251379678 & 61.37687098 \\
\hline 1.00242252 & 9.240019091 & 8.618974087 & 10.94573912 & 9.282412782 & 60.79533556 \\
\hline 1.014015914 & 7.496494068 & 12.63679079 & 10.97337412 & 6.360566234 & 61.40939982 \\
\hline 1.108905555 & 6.378048599 & 8.906360938 & 11.59024978 & 9.474361726 & 62.44178634 \\
\hline 0.965137688 & 8.344275429 & 8.259750897 & 14.39729182 & 7.842192946 & 60.07053401 \\
\hline 1.036886299 & 7.35839801 & 10.39663499 & 11.55555871 & 7.610252995 & 61.93559703 \\
\hline 1.02119965 & 7.663310877 & 7.655903139 & 17.17586544 & 5.293440686 & 61.07761478 \\
\hline 1.002978085 & 6.097955708 & 12.91017701 & 8.688207138 & 9.035115583 & 62.15936538 \\
\hline 1.056759543 & 6.655506809 & 13.29102632 & 11.97495408 & 5.284190519 & 61.63009152 \\
\hline 1.042340787 & 8.186617946 & 11.74004399 & 10.33668338 & 7.984174087 & 60.59649422 \\
\hline 1.088823315 & 8.56196082 & 10.30733901 & 8.916633311 & 8.966714833 & 62.05571281 \\
\hline 1.086817937 & 7.037782097 & 9.566742935 & 13.52392911 & 6.699472605 & 61.98069044 \\
\hline 1.060669253 & 7.922869518 & 7.325259047 & 16.4303006 & 5.941343908 & 61.20963583 \\
\hline 1.016706756 & 7.594923196 & 12.75443988 & 10.91525537 & 7.273034267 & 60.32834308 \\
\hline 1.09223416 & 7.071411188 & 7.906600289 & 16.48418815 & 5.694156821 & 61.64423809 \\
\hline 1.060667361 & 8.595859501 & 9.979065434 & 12.38383237 & 6.243405402 & 61.63131238 \\
\hline 1.09048865 & 9.046348284 & 10.24684007 & 9.97703616 & 8.711698186 & 60.81637027 \\
\hline 1.005616894 & 8.088718103 & 9.052308162 & 10.34413049 & 9.690667343 & 61.70896346 \\
\hline 1.076917829 & 6.902969424 & 9.781734405 & 11.7195776 & 8.214735713 & 62.20093406 \\
\hline 1.056562573 & 8.860536347 & 9.903427003 & 11.93668466 & 7.766716475 & 60.36138943 \\
\hline 1.022385609 & 6.699139449 & 7.814576274 & 12.55175983 & 8.879161303 & 62.93135445 \\
\hline 1.030562857 & 8.728609655 & 11.94669897 & 11.47854215 & 5.622440551 & 61.08144782 \\
\hline 1.065866087 & 9.849445405 & 9.784622465 & 11.96346426 & 7.039714844 & 60.18143086 \\
\hline 0.996281131 & 8.908356793 & 9.932541886 & 11.2980744 & 7.206715947 & 61.54792782 \\
\hline 1.070313514 & 8.97598003 & 9.843726711 & 11.33019023 & 7.393457302 & 61.27729651 \\
\hline 1.033263932 & 7.890259767 & 8.955704296 & 16.14161404 & 5.380789187 & 60.48109618 \\
\hline 1.066040356 & 8.601639737 & 9.250377252 & 12.25458692 & 7.041447152 & 61.68001465 \\
\hline 1.006176917 & 7.332667535 & 8.935538204 & 12.43652726 & 8.787808268 & 61.39041364 \\
\hline 1.026759573 & 9.247362252 & 7.94622405 & 14.69488613 & 5.86627534 & 61.10694431 \\
\hline 1.04692494 & 7.214817472 & 11.668321 & 13.69421085 & 4.959066795 & 61.30641808 \\
\hline 1.08680523 & 6.252067351 & 10.87426511 & 10.90082523 & 7.694049976 & 63.09443462 \\
\hline 1.05870926 & 6.897405909 & 8.783295169 & 17.9490028 & 4.917252109 & 60.2780199 \\
\hline 1.18237407 & 6.823501195 & 10.74918317 & 13.62214099 & 5.515837982 & 62.00713547 \\
\hline 1.033670553 & 8.808097534 & 11.69390639 & 10.9681638 & 7.520151985 & 59.85622293 \\
\hline 1.021810574 & 6.627677984 & 9.550778071 & 14.81917937 & 6.124179191 & 61.74740994 \\
\hline 1.069982242 & 9.12115061 & 7.494731696 & 13.10455019 & 7.274760225 & 61.82970546 \\
\hline 1.067145032 & 7.65346854 & 12.10564878 & 10.6827651 & 7.538834432 & 60.84148183 \\
\hline 1.061430281 & 8.400732028 & 7.630416791 & 10.94356447 & 9.5847253 & 62.27648283 \\
\hline 1.027866911 & 8.41593033 & 11.72740532 & 9.778042623 & 7.952320941 & 60.98748799 \\
\hline 1.072195178 & 7.888292967 & 9.238707596 & 10.8350439 & 9.290918311 & 61.56806037 \\
\hline 1.026151928 & 8.533766301 & 10.14352991 & 12.40361524 & 5.169856053 & 62.62029371 \\
\hline 1.075620368 & 6.805329762 & 12.50641063 & 12.73798389 & 5.392326207 & 61.37424256 \\
\hline
\end{tabular}


Table S5 shows the dependent variables for specific gravity of distillates calculated using the Improved Swing-Cut Method from data set 1.

Table S5: Dependent variables for specific gravity of distillates from data set 1

\begin{tabular}{|c|c|c|c|c|c|}
\hline $\mathrm{SG}_{\mathrm{LPG}}$ & $\mathrm{SG}_{\mathrm{N}}$ & $\mathrm{SG}_{\mathrm{K}}$ & $\mathrm{SG}_{\mathrm{LD}}$ & $\mathrm{SG}_{\mathrm{HD}}$ & $\mathrm{SG}_{\mathrm{ATR}}$ \\
\hline 0.54796633 & 0.735040789 & 0.815308475 & 0.863090005 & 0.901993516 & 0.986544204 \\
\hline 0.547453858 & 0.735038641 & 0.817945003 & 0.866821417 & 0.903237105 & 0.982389511 \\
\hline 0.545854201 & 0.73492886 & 0.823117631 & 0.867585969 & 0.900425871 & 0.980323092 \\
\hline 0.548496209 & 0.734931687 & 0.809006285 & 0.866094934 & 0.907466353 & 0.984194701 \\
\hline 0.5477253 & 0.72259269 & 0.799885584 & 0.856787041 & 0.896259566 & 0.981421099 \\
\hline 0.546498227 & 0.724667215 & 0.810593766 & 0.860981203 & 0.894060578 & 0.976554614 \\
\hline 0.548407103 & 0.745323105 & 0.822417424 & 0.869289582 & 0.909539017 & 0.987420366 \\
\hline 0.548929994 & 0.736673172 & 0.817894339 & 0.864776536 & 0.900025624 & 0.983396924 \\
\hline 0.547290726 & 0.731903043 & 0.8137004 & 0.865490586 & 0.902734354 & 0.981531182 \\
\hline 0.547596734 & 0.737524474 & 0.824793092 & 0.872923513 & 0.908821582 & 0.986399229 \\
\hline 0.548640405 & 0.726991934 & 0.804508601 & 0.863398798 & 0.90229476 & 0.981972713 \\
\hline 0.548615333 & 0.726665675 & 0.804982185 & 0.860022528 & 0.897522995 & 0.981133767 \\
\hline 0.544770604 & 0.725550471 & 0.804154603 & 0.854239613 & 0.890557673 & 0.972485391 \\
\hline 0.54828401 & 0.731785364 & 0.821400923 & 0.871846392 & 0.905993952 & 0.985530013 \\
\hline 0.546561389 & 0.730339553 & 0.808461412 & 0.860725282 & 0.901776395 & 0.982921325 \\
\hline 0.548469986 & 0.729434971 & 0.8163856 & 0.868633429 & 0.901619468 & 0.98080165 \\
\hline 0.545475297 & 0.707755579 & 0.789664605 & 0.854341093 & 0.891860883 & 0.972733599 \\
\hline 0.54532699 & 0.723306845 & 0.80615171 & 0.852249719 & 0.886176307 & 0.975606651 \\
\hline 0.547916108 & 0.735689358 & 0.823208482 & 0.87027003 & 0.904331538 & 0.983986773 \\
\hline 0.547638619 & 0.730676452 & 0.815482194 & 0.864462937 & 0.897441168 & 0.977457299 \\
\hline 0.546289533 & 0.720830397 & 0.806597614 & 0.859936967 & 0.894961009 & 0.978127885 \\
\hline 0.546962125 & 0.733507994 & 0.818207174 & 0.862842097 & 0.896150658 & 0.979288425 \\
\hline 0.548887323 & 0.729727549 & 0.811910794 & 0.862404101 & 0.896710057 & 0.981225691 \\
\hline 0.54655931 & 0.729869992 & 0.813577029 & 0.860159734 & 0.894976158 & 0.979593646 \\
\hline 0.546401851 & 0.738173968 & 0.817274334 & 0.8631616 & 0.902118814 & 0.981756683 \\
\hline 0.547583612 & 0.723303242 & 0.809615444 & 0.863538902 & 0.898599832 & 0.981383833 \\
\hline 0.546519641 & 0.720279644 & 0.800282925 & 0.855089327 & 0.892569117 & 0.977955392 \\
\hline 0.5456325 & 0.724401714 & 0.80232196 & 0.852295225 & 0.890007769 & 0.97676243 \\
\hline 0.547937209 & 0.728675157 & 0.81871446 & 0.867811645 & 0.90031967 & 0.982623675 \\
\hline 0.546635616 & 0.736258621 & 0.823321178 & 0.865391362 & 0.898482292 & 0.981669907 \\
\hline 0.547467475 & 0.726275804 & 0.817348852 & 0.863490218 & 0.8951283 & 0.982813033 \\
\hline 0.547466888 & 0.732807687 & 0.808174555 & 0.854048443 & 0.891080554 & 0.978668769 \\
\hline 0.547942657 & 0.739369156 & 0.816280304 & 0.865218165 & 0.906423467 & 0.985383914 \\
\hline 0.545580426 & 0.715496607 & 0.806117016 & 0.855048964 & 0.883419079 & 0.971642445 \\
\hline 0.547651264 & 0.724156308 & 0.799323004 & 0.855301642 & 0.89358317 & 0.977730297 \\
\hline 0.547946891 & 0.732699447 & 0.80692514 & 0.857024682 & 0.895681204 & 0.979459197 \\
\hline 0.547450107 & 0.719918 & 0.804331705 & 0.856232468 & 0.88955681 & 0.97773908 \\
\hline 0.547639107 & 0.730221679 & 0.807848393 & 0.85700626 & 0.893692172 & 0.979674029 \\
\hline 0.546909348 & 0.736156883 & 0.819098963 & 0.864001682 & 0.901520959 & 0.985137791 \\
\hline 0.547164066 & 0.745018866 & 0.827559158 & 0.871522563 & 0.911148194 & 0.9881885 \\
\hline 0.547151916 & 0.730038672 & 0.81070598 & 0.858594386 & 0.894447509 & 0.979614405 \\
\hline 0.547235238 & 0.727559044 & 0.814530138 & 0.859676142 & 0.891769117 & 0.980079586 \\
\hline 0.549084989 & 0.737820878 & 0.815567683 & 0.867133698 & 0.904626392 & 0.983061024 \\
\hline 0.545951664 & 0.716590272 & 0.810543801 & 0.865456313 & 0.896749215 & 0.976908812 \\
\hline 0.547329423 & 0.721309961 & 0.797216995 & 0.852665967 & 0.890584025 & 0.976892264 \\
\hline 0.548088939 & 0.731423985 & 0.815380651 & 0.86899503 & 0.90621069 & 0.984676612 \\
\hline 0.54739806 & 0.727874184 & 0.811551317 & 0.861524756 & 0.897265673 & 0.98171722 \\
\hline 0.54787807 & 0.715721458 & 0.805931458 & 0.865190636 & 0.89701702 & 0.976157061 \\
\hline 0.547169495 & 0.719399329 & 0.804467428 & 0.860314507 & 0.896029773 & 0.97980743 \\
\hline 0.547424882 & 0.7386593 & 0.825763114 & 0.867728051 & 0.900475451 & 0.982826267 \\
\hline 0.547919184 & 0.731926922 & 0.806868298 & 0.855830919 & 0.894179495 & 0.981503942 \\
\hline 0.547373022 & 0.734909057 & 0.811922238 & 0.856885599 & 0.893775774 & 0.979867168 \\
\hline 0.547213709 & 0.737262808 & 0.814967171 & 0.857845611 & 0.895312068 & 0.982345467 \\
\hline
\end{tabular}




\begin{tabular}{|c|c|c|c|c|c|}
\hline 0.5474217 & 0.730078962 & 0.812359872 & 0.863420578 & 0.90173145 & 0.984233344 \\
\hline 0.547883582 & 0.738444938 & 0.812638135 & 0.860827337 & 0.900059039 & 0.982725455 \\
\hline 0.547350909 & 0.731624017 & 0.804523353 & 0.855660339 & 0.894988669 & 0.978417044 \\
\hline 0.54851952 & 0.734231309 & 0.813196919 & 0.866010263 & 0.905047625 & 0.985526596 \\
\hline 0.54704078 & 0.722816675 & 0.808755073 & 0.865219367 & 0.900325025 & 0.97886485 \\
\hline 0.547766088 & 0.74114883 & 0.813871964 & 0.859843782 & 0.901635446 & 0.98434192 \\
\hline 0.54743158 & 0.732664506 & 0.812642011 & 0.85612669 & 0.890150672 & 0.97808528 \\
\hline 0.546107375 & 0.722321351 & 0.814785199 & 0.863472661 & 0.894467335 & 0.978109912 \\
\hline 0.547568967 & 0.730974051 & 0.811314607 & 0.860258986 & 0.899013684 & 0.985601287 \\
\hline 0.546938072 & 0.721760616 & 0.798157978 & 0.852294795 & 0.888954911 & 0.974875315 \\
\hline 0.547736903 & 0.730112478 & 0.814584468 & 0.862507497 & 0.896705639 & 0.981210642 \\
\hline 0.547585762 & 0.727238402 & 0.801604746 & 0.859831368 & 0.900179525 & 0.97954367 \\
\hline 0.54730344 & 0.718740746 & 0.811557921 & 0.859733068 & 0.889673481 & 0.979022905 \\
\hline 0.547405903 & 0.725321692 & 0.816932223 & 0.870286917 & 0.90309608 & 0.981879924 \\
\hline 0.546382372 & 0.726574878 & 0.814418517 & 0.861835323 & 0.894880276 & 0.979395543 \\
\hline 0.546846032 & 0.738634094 & 0.825045565 & 0.863976019 & 0.897115877 & 0.983537433 \\
\hline 0.54795332 & 0.733068978 & 0.814017444 & 0.865685515 & 0.904000052 & 0.984366444 \\
\hline 0.547325887 & 0.731301659 & 0.805687753 & 0.860771865 & 0.902328068 & 0.981749706 \\
\hline 0.547228414 & 0.723136696 & 0.811540365 & 0.863152584 & 0.895545119 & 0.978410753 \\
\hline 0.548576958 & 0.734772581 & 0.808009524 & 0.865533067 & 0.908070999 & 0.985035704 \\
\hline 0.546021994 & 0.732612237 & 0.818956062 & 0.864308346 & 0.899017923 & 0.980890532 \\
\hline 0.547335813 & 0.738104278 & 0.821436075 & 0.864357503 & 0.899626972 & 0.983257663 \\
\hline 0.547925822 & 0.731495747 & 0.812454535 & 0.856467458 & 0.889993031 & 0.979367464 \\
\hline 0.54755123 & 0.730810431 & 0.813863732 & 0.862269617 & 0.898860052 & 0.983570134 \\
\hline 0.546510376 & 0.730786096 & 0.813461547 & 0.860997561 & 0.897140726 & 0.980176563 \\
\hline 0.548473402 & 0.731078065 & 0.808114301 & 0.856861931 & 0.893407158 & 0.981664976 \\
\hline 0.547036288 & 0.732165447 & 0.821216232 & 0.86777956 & 0.899483231 & 0.979628047 \\
\hline 0.546521739 & 0.735906549 & 0.818842649 & 0.863989065 & 0.89980577 & 0.980607918 \\
\hline 0.546999969 & 0.732135728 & 0.817519795 & 0.860698575 & 0.892476856 & 0.977929524 \\
\hline 0.547275985 & 0.737841881 & 0.821254302 & 0.865017399 & 0.900109779 & 0.982321047 \\
\hline 0.548843988 & 0.73119776 & 0.806316561 & 0.864339829 & 0.903915895 & 0.980928479 \\
\hline 0.546573321 & 0.734890714 & 0.818503213 & 0.863106473 & 0.898851461 & 0.981721914 \\
\hline 0.547171162 & 0.723929457 & 0.803800358 & 0.854748765 & 0.890677402 & 0.978534824 \\
\hline 0.546892866 & 0.734356246 & 0.813472028 & 0.861355264 & 0.898328598 & 0.979299057 \\
\hline 0.547685017 & 0.727761366 & 0.814049069 & 0.868649877 & 0.903488401 & 0.981320031 \\
\hline 0.548073535 & 0.732800186 & 0.819646272 & 0.866531532 & 0.901292872 & 0.985174263 \\
\hline 0.547650491 & 0.723558613 & 0.797361529 & 0.862386104 & 0.905503559 & 0.981268589 \\
\hline 0.547380104 & 0.736674454 & 0.822309805 & 0.874995588 & 0.915243171 & 0.989445949 \\
\hline 0.547392338 & 0.729682133 & 0.815000672 & 0.863701752 & 0.897358428 & 0.979218314 \\
\hline 0.54820186 & 0.725449125 & 0.805162525 & 0.861923347 & 0.899162557 & 0.980585743 \\
\hline 0.546907489 & 0.739791786 & 0.818752857 & 0.861656246 & 0.899257802 & 0.982348929 \\
\hline 0.546032727 & 0.724943874 & 0.814450916 & 0.863447369 & 0.897458911 & 0.980716315 \\
\hline 0.546941517 & 0.736884339 & 0.816604 & 0.857266921 & 0.893240995 & 0.982184967 \\
\hline 0.545585637 & 0.725835554 & 0.815968149 & 0.860507508 & 0.891849157 & 0.978148085 \\
\hline 0.546980519 & 0.732079824 & 0.81404134 & 0.85924871 & 0.895391102 & 0.982365572 \\
\hline 0.547800964 & 0.738956123 & 0.824990645 & 0.868165692 & 0.900267509 & 0.981110555 \\
\hline 0.547174623 & 0.726035122 & 0.815209843 & 0.869703672 & 0.904571041 & 0.982608627 \\
\hline
\end{tabular}


Table S6 shows the dependent variables for sulfur content of distillates calculated using the Improved Swing-Cut Method from data set 1.

Table S6: Dependent variables for sulfur content of distillates from data set 1

\begin{tabular}{|c|c|c|c|c|}
\hline $\mathrm{S}_{\mathrm{N}}$ & $\mathrm{S}_{\mathrm{K}}$ & $\mathrm{S}_{\mathrm{LD}}$ & $\mathrm{S}_{\mathrm{HD}}$ & $\mathrm{S}_{\mathrm{ATR}}$ \\
\hline 0.004411258 & 0.078810801 & 0.249719462 & 0.491839154 & 0.78147386 \\
\hline 0.007436213 & 0.087007962 & 0.268075388 & 0.475345327 & 0.739370276 \\
\hline 0.008048212 & 0.094645078 & 0.296470277 & 0.466698609 & 0.762040219 \\
\hline 0.005636645 & 0.071346755 & 0.270279363 & 0.51015702 & 0.752821246 \\
\hline 0.00300331 & 0.059605458 & 0.208251784 & 0.442352556 & 0.729528048 \\
\hline 0.004822081 & 0.074093413 & 0.243294514 & 0.437838811 & 0.724537368 \\
\hline 0.009459914 & 0.092198101 & 0.283068562 & 0.517050133 & 0.769621917 \\
\hline 0.006594057 & 0.088880619 & 0.253403561 & 0.476643144 & 0.74549309 \\
\hline 0.006235428 & 0.079281248 & 0.265361142 & 0.475686143 & 0.74080486 \\
\hline 0.006372269 & 0.099945239 & 0.30846614 & 0.505994318 & 0.767923818 \\
\hline 0.003547224 & 0.06692128 & 0.25143622 & 0.486861917 & 0.737981524 \\
\hline 0.003985141 & 0.068756695 & 0.221470184 & 0.448987819 & 0.71837724 \\
\hline 0.005649054 & 0.059581977 & 0.219636098 & 0.412095453 & 0.718610188 \\
\hline 0.003927444 & 0.09736525 & 0.297966857 & 0.497585627 & 0.75647206 \\
\hline 0.005195229 & 0.067238545 & 0.244611793 & 0.475545133 & 0.764181853 \\
\hline 0.004536571 & 0.087195172 & 0.290413537 & 0.49255209 & 0.744140835 \\
\hline 0.001865701 & 0.043420762 & 0.219253192 & 0.421404348 & 0.7137375 \\
\hline 0.004525268 & 0.063803765 & 0.20115814 & 0.412227188 & 0.744672514 \\
\hline 0.006820974 & 0.099037768 & 0.285646359 & 0.48252207 & 0.743968491 \\
\hline 0.006725762 & 0.083037516 & 0.254782948 & 0.451629071 & 0.710800511 \\
\hline 0.003386272 & 0.067247057 & 0.245136454 & 0.450240929 & 0.747027769 \\
\hline 0.007474034 & 0.08663270 & 0.251917593 & 0.451742497 & 0.738672366 \\
\hline 0.00428800 & 0.07916523 & 0.246072326 & 0.469892773 & 0.744560861 \\
\hline 0.006277626 & 0.07845601 & 0.231553829 & 0.438170273 & 0.735156002 \\
\hline 0.009979598 & 0.082568147 & 0.248121276 & 0.459108473 & 0.738078325 \\
\hline 0.002958545 & 0.074420166 & 0.258083378 & 0.472073887 & 0.753130184 \\
\hline 0.003506506 & 0.058632268 & 0.204638711 & 0.424019278 & 0.722782489 \\
\hline 0.005013108 & 0.058731433 & 0.196902022 & 0.417117603 & 0.732599819 \\
\hline 0.003794354 & 0.091121588 & 0.288675912 & 0.487973019 & 0.76495521 \\
\hline 0.008293995 & 0.096127027 & 0.267038856 & 0.460223571 & 0.753740444 \\
\hline 0.003350586 & 0.088572345 & 0.252395751 & 0.455592627 & 0.762092904 \\
\hline 0.006968032 & 0.068955924 & 0.201154186 & 0.432080309 & 0.734853605 \\
\hline 0.008789816 & 0.083757888 & 0.245721846 & 0.475302009 & 0.730887669 \\
\hline 0.002947031 & 0.066439936 & 0.206604477 & 0.38689441 & 0.705178995 \\
\hline 0.003968902 & 0.057104217 & 0.212639315 & 0.446438002 & 0.728679575 \\
\hline 0.006817306 & 0.068126282 & 0.215710114 & 0.449812684 & 0.727384901 \\
\hline 0.002904671 & 0.065865366 & 0.212989233 & 0.429059719 & 0.735139079 \\
\hline 0.004999775 & 0.067974849 & 0.229354272 & 0.464377758 & 0.761169682 \\
\hline 0.007551604 & 0.087926283 & 0.24005179 & 0.453962382 & 0.742491421 \\
\hline 0.010679277 & 0.101501097 & 0.288542436 & 0.499767102 & 0.763595827 \\
\hline 0.005772092 & 0.073541495 & 0.227814943 & 0.448085942 & 0.743038418 \\
\hline 0.00454715 & 0.081591506 & 0.233217023 & 0.442091859 & 0.75290697 \\
\hline 0.007172629 & 0.08404237 & 0.273393838 & 0.504055442 & 0.744667522 \\
\hline 0.002951201 & 0.076720014 & 0.268118764 & 0.437367825 & 0.721317403 \\
\hline 0.00378422 & 0.055243157 & 0.188679311 & 0.412163138 & 0.705381603 \\
\hline 0.004357964 & 0.084226576 & 0.280096125 & 0.495462802 & 0.750832532 \\
\hline 0.004433617 & 0.076632007 & 0.238719474 & 0.456280839 & 0.745737692 \\
\hline 0.002425886 & 0.071192668 & 0.260531749 & 0.453347387 & 0.703581018 \\
\hline 0.002540394 & 0.065276423 & 0.243767953 & 0.461973849 & 0.751938626 \\
\hline 0.008429633 & 0.101977641 & 0.283786107 & 0.478653056 & 0.761093183 \\
\hline 0.005276866 & 0.066604655 & 0.214324156 & 0.459353963 & 0.760177564 \\
\hline 0.007631097 & 0.074650888 & 0.217610221 & 0.446244075 & 0.743549355 \\
\hline 0.007949957 & 0.078490526 & 0.22239071 & 0.456173947 & 0.761979954 \\
\hline
\end{tabular}




\begin{tabular}{|c|c|c|c|c|}
\hline 0.004537416 & 0.077687155 & 0.242873805 & 0.465835902 & 0.745778934 \\
\hline 0.007044075 & 0.073415447 & 0.256719956 & 0.500031888 & 0.782700088 \\
\hline 0.006448346 & 0.06295575 & 0.218186354 & 0.454424672 & 0.738110261 \\
\hline 0.004309746 & 0.077617174 & 0.270543463 & 0.508380865 & 0.771400686 \\
\hline 0.003895143 & 0.07327659 & 0.261979925 & 0.45858863 & 0.721818937 \\
\hline 0.009740735 & 0.077527917 & 0.225707335 & 0.469537324 & 0.747125911 \\
\hline 0.007066731 & 0.076751845 & 0.209515228 & 0.426203465 & 0.730521252 \\
\hline 0.003733428 & 0.082020124 & 0.270636335 & 0.451371761 & 0.753786105 \\
\hline 0.003764614 & 0.072890603 & 0.229983651 & 0.471056539 & 0.772178641 \\
\hline 0.00390263 & 0.054705033 & 0.196036932 & 0.41881579 & 0.714657101 \\
\hline 0.004892805 & 0.081603519 & 0.252508821 & 0.467890181 & 0.75580581 \\
\hline 0.004651979 & 0.059921034 & 0.241225045 & 0.474335714 & 0.737035716 \\
\hline 0.002343201 & 0.07843971 & 0.242134457 & 0.444554848 & 0.761370191 \\
\hline 0.003465849 & 0.088406424 & 0.301675111 & 0.486276841 & 0.752704892 \\
\hline 0.005129148 & 0.081079863 & 0.241692108 & 0.437424075 & 0.735980065 \\
\hline 0.008504782 & 0.098836595 & 0.256514181 & 0.460862403 & 0.767872303 \\
\hline 0.005045393 & 0.079815843 & 0.264900852 & 0.491406442 & 0.758315537 \\
\hline 0.005820659 & 0.065159505 & 0.241673295 & 0.476398439 & 0.744318062 \\
\hline 0.003950513 & 0.078304687 & 0.247234299 & 0.442591679 & 0.722279712 \\
\hline 0.00536381 & 0.070376248 & 0.260428971 & 0.505465164 & 0.74694303 \\
\hline 0.006886098 & 0.086244515 & 0.270666938 & 0.46416546 & 0.762597679 \\
\hline 0.00899898 & 0.093041836 & 0.245412813 & 0.453020585 & 0.737514305 \\
\hline 0.005692476 & 0.077230865 & 0.214317447 & 0.437589037 & 0.747702767 \\
\hline 0.004518355 & 0.078938238 & 0.247061935 & 0.472974757 & 0.765241571 \\
\hline 0.006729771 & 0.078146946 & 0.233668601 & 0.441101826 & 0.730831201 \\
\hline 0.004240159 & 0.068986401 & 0.223783154 & 0.469035025 & 0.771238522 \\
\hline 0.006745433 & 0.093621538 & 0.286470249 & 0.46729828 & 0.741467258 \\
\hline 0.00919452 & 0.087000522 & 0.248634396 & 0.447699232 & 0.727354144 \\
\hline 0.006455914 & 0.085073804 & 0.249534382 & 0.450706046 & 0.74966158 \\
\hline 0.008763006 & 0.092182416 & 0.259101877 & 0.466035902 & 0.746789831 \\
\hline 0.005666673 & 0.069896976 & 0.251004157 & 0.484702506 & 0.715783254 \\
\hline 0.007474459 & 0.085438725 & 0.257448111 & 0.465785954 & 0.760189341 \\
\hline 0.003773305 & 0.062873295 & 0.209367078 & 0.437462805 & 0.745740191 \\
\hline 0.007755376 & 0.076830488 & 0.249609824 & 0.46316404 & 0.742746213 \\
\hline 0.00436595 & 0.082171006 & 0.287660197 & 0.486474161 & 0.743071962 \\
\hline 0.004000269 & 0.090149009 & 0.277549769 & 0.49532812 & 0.780936581 \\
\hline 0.003420822 & 0.056017058 & 0.24455896 & 0.477424401 & 0.721045488 \\
\hline 0.004690323 & 0.092669264 & 0.309598181 & 0.516645074 & 0.768947238 \\
\hline 0.006323091 & 0.083135416 & 0.242379316 & 0.440084123 & 0.711476177 \\
\hline 0.003423798 & 0.066737085 & 0.251670286 & 0.481468008 & 0.747687768 \\
\hline 0.009493388 & 0.084694984 & 0.247588223 & 0.4697824 & 0.761349578 \\
\hline 0.004366868 & 0.081086762 & 0.253036151 & 0.446804182 & 0.745730034 \\
\hline 0.007706498 & 0.080716698 & 0.221039614 & 0.451779734 & 0.770087019 \\
\hline 0.005129455 & 0.082458119 & 0.244223766 & 0.431892253 & 0.749077181 \\
\hline 0.005969182 & 0.078463256 & 0.226075189 & 0.448970163 & 0.755335413 \\
\hline 0.008067145 & 0.100215821 & 0.303547812 & 0.496050792 & 0.769344319 \\
\hline 0.003661800 & 0.084293562 & 0.29233006 & 0.483998843 & 0.74997722 \\
\hline
\end{tabular}




\section{Appendix C - Average Errors for each Surrogate Model}

The least squares regression errors of each distillation component for each surrogate model are shown in Tables S7 to S10. Each error corresponds to the average error from the 100 points in the data set.

Table S7: Least Squares errors for Data Set 1 using the CSW model

\begin{tabular}{|c|c|c|c|c|c|c|c|c|c|c|}
\hline & Model 1 & Model 2 & Model 3 & Model 4 & Model 5 & Model 6 & Model 7 & Model 8 & Model 9 & $\begin{array}{c}\text { Model } \\
10 \\
\end{array}$ \\
\hline $\mathrm{YLD}_{\mathrm{LPG}}$ & $4.83 \mathrm{E}-30$ & $4.83 \mathrm{E}-30$ & $4.83 \mathrm{E}-30$ & $4.83 \mathrm{E}-30$ & $1.68 \mathrm{E}-29$ & $1.68 \mathrm{E}-29$ & $1.68 \mathrm{E}-29$ & $1.68 \mathrm{E}-29$ & 4.43E-30 & 4.43E-30 \\
\hline $\mathrm{YLD}_{\mathrm{N}}$ & $6.90 \mathrm{E}-01$ & $2.85 \mathrm{E}-03$ & $3.21 \mathrm{E}-16$ & $3.21 \mathrm{E}-16$ & $2.85 \mathrm{E}-03$ & $6.90 \mathrm{E}-01$ & $3.21 \mathrm{E}-16$ & $1.14 \mathrm{E}-02$ & $3.21 \mathrm{E}-16$ & $3.21 \mathrm{E}-16$ \\
\hline $\mathrm{YLD}_{\mathrm{K}}$ & $3.25 \mathrm{E}+00$ & $6.61 \mathrm{E}-03$ & $1.62 \mathrm{E}-15$ & $1.62 \mathrm{E}-15$ & $6.61 \mathrm{E}-03$ & $3.25 \mathrm{E}+00$ & $1.62 \mathrm{E}-15$ & $7.66 \mathrm{E}-02$ & $1.62 \mathrm{E}-15$ & $1.62 \mathrm{E}-15$ \\
\hline $\mathrm{YLD}_{\mathrm{LD}}$ & $3.65 \mathrm{E}+00$ & $3.75 \mathrm{E}-03$ & $1.09 \mathrm{E}-15$ & $1.09 \mathrm{E}-15$ & $3.75 \mathrm{E}-03$ & $3.65 \mathrm{E}+00$ & $1.09 \mathrm{E}-15$ & $1.14 \mathrm{E}-01$ & $1.09 \mathrm{E}-15$ & $1.09 \mathrm{E}-15$ \\
\hline $\mathrm{YLD}_{\mathrm{HD}}$ & $1.84 \mathrm{E}+00$ & $1.13 \mathrm{E}-04$ & $3.38 \mathrm{E}-16$ & $3.38 \mathrm{E}-16$ & $2.95 \mathrm{E}-03$ & $1.84 \mathrm{E}+00$ & $1.19 \mathrm{E}-03$ & $6.13 \mathrm{E}-02$ & $3.38 \mathrm{E}-16$ & $3.38 \mathrm{E}-16$ \\
\hline $\mathrm{YLD}_{\mathrm{ATR}}$ & $2.62 \mathrm{E}-17$ & $2.62 \mathrm{E}-17$ & $2.62 \mathrm{E}-17$ & $2.62 \mathrm{E}-17$ & $1.37 \mathrm{E}-01$ & $1.37 \mathrm{E}-01$ & $1.37 \mathrm{E}-01$ & $1.37 \mathrm{E}-01$ & $2.62 \mathrm{E}-17$ & $2.62 \mathrm{E}-17$ \\
\hline $\mathrm{SG}_{\mathrm{LPG}}$ & $1.29 \mathrm{E}-09$ & $1.29 \mathrm{E}-09$ & $1.29 \mathrm{E}-09$ & $1.29 \mathrm{E}-09$ & $5.32 \mathrm{E}-08$ & $5.32 \mathrm{E}-08$ & $5.32 \mathrm{E}-08$ & $5.32 \mathrm{E}-08$ & 1.29E-09 & $1.29 \mathrm{E}-09$ \\
\hline $\mathrm{SG}_{\mathrm{N}}$ & $2.14 \mathrm{E}-05$ & $3.09 \mathrm{E}-07$ & $2.82 \mathrm{E}-07$ & $2.82 \mathrm{E}-07$ & $3.09 \mathrm{E}-07$ & $2.14 \mathrm{E}-05$ & $2.82 \mathrm{E}-07$ & $8.35 \mathrm{E}-07$ & $2.82 \mathrm{E}-07$ & $2.82 \mathrm{E}-07$ \\
\hline $\mathrm{SG}_{\mathrm{K}}$ & $3.01 \mathrm{E}-05$ & $8.00 \mathrm{E}-07$ & $5.23 \mathrm{E}-07$ & $5.23 \mathrm{E}-07$ & $8.00 \mathrm{E}-07$ & $3.01 \mathrm{E}-05$ & $5.23 \mathrm{E}-07$ & $1.25 \mathrm{E}-06$ & $5.23 \mathrm{E}-07$ & $5.23 \mathrm{E}-07$ \\
\hline $\mathrm{SG}_{\mathrm{LD}}$ & $1.42 \mathrm{E}-05$ & $1.55 \mathrm{E}-07$ & 1.17E-07 & 1.17E-07 & $1.55 \mathrm{E}-07$ & $1.42 \mathrm{E}-05$ & $1.17 \mathrm{E}-07$ & 4.41E-07 & $1.17 \mathrm{E}-07$ & $1.17 \mathrm{E}-07$ \\
\hline $\mathrm{SG}_{\mathrm{HD}}$ & $7.40 \mathrm{E}-06$ & $2.21 \mathrm{E}-07$ & $2.16 \mathrm{E}-07$ & $2.16 \mathrm{E}-07$ & $2.21 \mathrm{E}-07$ & $7.40 \mathrm{E}-06$ & $2.16 \mathrm{E}-07$ & $4.01 \mathrm{E}-07$ & $2.16 \mathrm{E}-07$ & $2.16 \mathrm{E}-07$ \\
\hline $\mathrm{SG}_{\mathrm{ATR}}$ & $2.70 \mathrm{E}-09$ & $2.70 \mathrm{E}-09$ & $2.70 \mathrm{E}-09$ & $2.70 \mathrm{E}-09$ & $2.70 \mathrm{E}-09$ & $2.70 \mathrm{E}-09$ & $2.70 \mathrm{E}-09$ & $2.70 \mathrm{E}-09$ & $2.70 \mathrm{E}-09$ & $2.70 \mathrm{E}-09$ \\
\hline $\mathrm{S}_{\mathrm{N}}$ & $3.17 \mathrm{E}-06$ & $1.82 \mathrm{E}-07$ & $3.39 \mathrm{E}-08$ & 3.39E-08 & $1.82 \mathrm{E}-07$ & $3.17 \mathrm{E}-06$ & $3.39 \mathrm{E}-08$ & $1.61 \mathrm{E}-07$ & 3.39E-08 & $3.39 \mathrm{E}-08$ \\
\hline $\mathrm{S}_{\mathrm{K}}$ & $8.98 \mathrm{E}-05$ & $2.10 \mathrm{E}-06$ & $1.76 \mathrm{E}-06$ & $1.76 \mathrm{E}-06$ & $2.10 \mathrm{E}-06$ & $8.98 \mathrm{E}-05$ & $1.76 \mathrm{E}-06$ & $5.43 \mathrm{E}-06$ & $1.76 \mathrm{E}-06$ & $1.76 \mathrm{E}-06$ \\
\hline $\mathrm{S}_{\mathrm{LD}}$ & $5.55 \mathrm{E}-04$ & $6.37 \mathrm{E}-06$ & 4.49E-06 & 4.49E-06 & $6.37 \mathrm{E}-06$ & $5.55 \mathrm{E}-04$ & 4.49E-06 & $2.44 \mathrm{E}-05$ & 4.49E-06 & 4.49E-06 \\
\hline $\mathrm{S}_{\mathrm{HD}}$ & $1.46 \mathrm{E}-04$ & $6.31 \mathrm{E}-06$ & $4.05 \mathrm{E}-06$ & $4.05 \mathrm{E}-06$ & $6.69 \mathrm{E}-06$ & $1.62 \mathrm{E}-04$ & $4.05 \mathrm{E}-06$ & $2.71 \mathrm{E}-05$ & 4.05E-06 & $4.05 \mathrm{E}-06$ \\
\hline $\mathrm{S}_{\mathrm{ATR}}$ & $1.23 \mathrm{E}-07$ & $1.23 \mathrm{E}-07$ & $1.23 \mathrm{E}-07$ & $1.23 \mathrm{E}-07$ & $3.15 \mathrm{E}-05$ & $3.15 \mathrm{E}-05$ & $3.15 \mathrm{E}-05$ & $3.15 \mathrm{E}-05$ & $1.23 \mathrm{E}-07$ & $1.23 \mathrm{E}-07$ \\
\hline
\end{tabular}


Table S8: Least Squares errors for Data Set 2 using the CSW model

\begin{tabular}{|c|c|c|c|c|c|c|c|c|c|c|}
\hline & Model 1 & Model 2 & Model 3 & Model 4 & Model 5 & Model 6 & Model 7 & Model 8 & Model 9 & $\begin{array}{c}\text { Model } \\
10\end{array}$ \\
\hline YLD $_{\mathrm{LPG}}$ & $1.50 \mathrm{E}-29$ & $1.50 \mathrm{E}-29$ & $1.50 \mathrm{E}-29$ & $1.50 \mathrm{E}-29$ & 2.39E-29 & $2.39 \mathrm{E}-29$ & 2.39E-29 & $2.39 \mathrm{E}-29$ & $1.50 \mathrm{E}-29$ & $1.50 \mathrm{E}-29$ \\
\hline $\mathrm{YLD}_{\mathrm{N}}$ & $8.20 \mathrm{E}-01$ & $2.18 \mathrm{E}-03$ & $7.03 \mathrm{E}-16$ & $7.03 \mathrm{E}-16$ & $2.18 \mathrm{E}-03$ & $8.20 \mathrm{E}-01$ & $7.03 \mathrm{E}-16$ & $9.63 \mathrm{E}-03$ & $7.03 \mathrm{E}-16$ & $7.03 \mathrm{E}-16$ \\
\hline $\mathrm{YLD}_{\mathrm{K}}$ & $3.31 \mathrm{E}+00$ & $5.76 \mathrm{E}-03$ & $1.60 \mathrm{E}-15$ & $1.60 \mathrm{E}-15$ & $5.76 \mathrm{E}-03$ & $3.31 \mathrm{E}+00$ & $1.60 \mathrm{E}-15$ & $7.38 \mathrm{E}-02$ & $1.60 \mathrm{E}-15$ & $1.60 \mathrm{E}-15$ \\
\hline $\mathrm{YLD}_{\mathrm{LD}}$ & $4.82 \mathrm{E}+00$ & $2.56 \mathrm{E}-03$ & $1.46 \mathrm{E}-15$ & $1.46 \mathrm{E}-15$ & $2.56 \mathrm{E}-03$ & $4.82 \mathrm{E}+00$ & $1.46 \mathrm{E}-15$ & $1.23 \mathrm{E}-01$ & $1.46 \mathrm{E}-15$ & $1.46 \mathrm{E}-15$ \\
\hline $\mathrm{YLD}_{\mathrm{HD}}$ & $1.92 \mathrm{E}+00$ & $1.44 \mathrm{E}-04$ & $4.04 \mathrm{E}-16$ & $4.04 \mathrm{E}-16$ & $3.14 \mathrm{E}-03$ & $1.92 \mathrm{E}+00$ & $1.88 \mathrm{E}-03$ & $7.59 \mathrm{E}-02$ & $4.04 \mathrm{E}-16$ & $4.04 \mathrm{E}-16$ \\
\hline $\mathrm{YLD}_{\mathrm{ATR}}$ & $8.32 \mathrm{E}-16$ & $8.32 \mathrm{E}-16$ & $8.32 \mathrm{E}-16$ & $8.32 \mathrm{E}-16$ & $1.22 \mathrm{E}-01$ & $1.22 \mathrm{E}-01$ & $1.22 \mathrm{E}-01$ & $1.22 \mathrm{E}-01$ & $8.32 \mathrm{E}-16$ & $8.32 \mathrm{E}-16$ \\
\hline $\mathrm{SG}_{\mathrm{LPG}}$ & $1.73 \mathrm{E}-09$ & $1.73 \mathrm{E}-09$ & $1.73 \mathrm{E}-09$ & $1.73 \mathrm{E}-09$ & 4.47E-08 & 4.47E-08 & 4.47E-08 & $4.47 \mathrm{E}-08$ & $1.73 \mathrm{E}-09$ & $1.73 \mathrm{E}-09$ \\
\hline $\mathrm{SG}_{\mathrm{N}}$ & $2.60 \mathrm{E}-05$ & 4.47E-07 & $3.65 \mathrm{E}-07$ & $3.65 \mathrm{E}-07$ & 4.47E-07 & $2.60 \mathrm{E}-05$ & $3.65 \mathrm{E}-07$ & $6.43 \mathrm{E}-07$ & $3.65 \mathrm{E}-07$ & $3.65 \mathrm{E}-07$ \\
\hline $\mathrm{SG}_{\mathrm{K}}$ & $3.95 \mathrm{E}-05$ & $8.18 \mathrm{E}-07$ & $8.42 \mathrm{E}-07$ & $8.42 \mathrm{E}-07$ & $8.18 \mathrm{E}-07$ & $3.95 \mathrm{E}-05$ & $8.42 \mathrm{E}-07$ & $1.45 \mathrm{E}-06$ & $8.42 \mathrm{E}-07$ & $8.42 \mathrm{E}-07$ \\
\hline $\mathrm{SG}_{\mathrm{LD}}$ & $1.27 \mathrm{E}-05$ & $1.78 \mathrm{E}-07$ & $1.85 \mathrm{E}-07$ & $1.85 \mathrm{E}-07$ & $1.78 \mathrm{E}-07$ & $1.27 \mathrm{E}-05$ & $1.85 \mathrm{E}-07$ & $6.34 \mathrm{E}-07$ & $1.85 \mathrm{E}-07$ & $1.85 \mathrm{E}-07$ \\
\hline $\mathrm{SG}_{\mathrm{HD}}$ & 8.37E-06 & $2.75 \mathrm{E}-07$ & $2.74 \mathrm{E}-07$ & $2.74 \mathrm{E}-07$ & $2.75 \mathrm{E}-07$ & 8.37E-06 & $2.74 \mathrm{E}-07$ & $4.52 \mathrm{E}-07$ & $2.74 \mathrm{E}-07$ & $2.74 \mathrm{E}-07$ \\
\hline $\mathrm{SG}_{\mathrm{ATR}}$ & 1.93E-09 & $1.93 \mathrm{E}-09$ & $1.93 \mathrm{E}-09$ & $1.93 \mathrm{E}-09$ & $1.93 \mathrm{E}-09$ & $1.93 \mathrm{E}-09$ & $1.93 \mathrm{E}-09$ & $1.93 \mathrm{E}-09$ & $1.93 \mathrm{E}-09$ & $1.93 \mathrm{E}-09$ \\
\hline $\mathrm{S}_{\mathrm{N}}$ & 3.69E-06 & $1.66 \mathrm{E}-07$ & $4.42 \mathrm{E}-08$ & $4.42 \mathrm{E}-08$ & $1.66 \mathrm{E}-07$ & $3.69 \mathrm{E}-06$ & $4.42 \mathrm{E}-08$ & $1.22 \mathrm{E}-07$ & $4.42 \mathrm{E}-08$ & $4.42 \mathrm{E}-08$ \\
\hline $\mathrm{S}_{\mathrm{K}}$ & $1.19 \mathrm{E}-04$ & $2.76 \mathrm{E}-06$ & $2.82 \mathrm{E}-06$ & $2.82 \mathrm{E}-06$ & $2.76 \mathrm{E}-06$ & $1.19 \mathrm{E}-04$ & $2.82 \mathrm{E}-06$ & $6.11 \mathrm{E}-06$ & $2.82 \mathrm{E}-06$ & $2.82 \mathrm{E}-06$ \\
\hline $\mathrm{S}_{\mathrm{LD}}$ & 4.88E-04 & $6.79 \mathrm{E}-06$ & $7.06 \mathrm{E}-06$ & $7.06 \mathrm{E}-06$ & $6.79 \mathrm{E}-06$ & $4.88 \mathrm{E}-04$ & $7.06 \mathrm{E}-06$ & $3.50 \mathrm{E}-05$ & $7.06 \mathrm{E}-06$ & $7.06 \mathrm{E}-06$ \\
\hline $\mathrm{S}_{\mathrm{HD}}$ & $1.63 \mathrm{E}-04$ & $6.67 \mathrm{E}-06$ & $5.19 \mathrm{E}-06$ & $5.19 \mathrm{E}-06$ & $5.98 \mathrm{E}-06$ & $1.57 \mathrm{E}-04$ & 5.19E-06 & $1.82 \mathrm{E}-05$ & $5.19 \mathrm{E}-06$ & $5.19 \mathrm{E}-06$ \\
\hline $\mathrm{S}_{\mathrm{ATR}}$ & $1.13 \mathrm{E}-07$ & $1.13 \mathrm{E}-07$ & $1.13 \mathrm{E}-07$ & $1.13 \mathrm{E}-07$ & $2.76 \mathrm{E}-05$ & $2.76 \mathrm{E}-05$ & $2.76 \mathrm{E}-05$ & $2.76 \mathrm{E}-05$ & $1.13 \mathrm{E}-07$ & $1.13 \mathrm{E}-07$ \\
\hline
\end{tabular}


Table S9: Least Squares errors for Data Set 1 using the ISW model

\begin{tabular}{|c|c|c|c|c|c|c|c|c|c|c|}
\hline & Model 1 & Model 2 & Model 3 & Model 4 & Model 5 & Model 6 & Model 7 & Model 8 & Model 9 & $\begin{array}{c}\text { Model } \\
10\end{array}$ \\
\hline YLD $_{\mathrm{LPG}}$ & $4.83 \mathrm{E}-30$ & $4.83 \mathrm{E}-30$ & $4.83 \mathrm{E}-30$ & $4.83 \mathrm{E}-30$ & $1.68 \mathrm{E}-29$ & $1.68 \mathrm{E}-29$ & $1.68 \mathrm{E}-29$ & $1.68 \mathrm{E}-29$ & $4.43 \mathrm{E}-30$ & $4.43 \mathrm{E}-30$ \\
\hline $\mathrm{YLD}_{\mathrm{N}}$ & $6.90 \mathrm{E}-01$ & $2.85 \mathrm{E}-03$ & $3.21 \mathrm{E}-16$ & $3.21 \mathrm{E}-16$ & $2.85 \mathrm{E}-03$ & $6.90 \mathrm{E}-01$ & $3.21 \mathrm{E}-16$ & $1.14 \mathrm{E}-02$ & $3.21 \mathrm{E}-16$ & $3.21 \mathrm{E}-16$ \\
\hline $\mathrm{YLD}_{\mathrm{K}}$ & $3.25 \mathrm{E}+00$ & $6.61 \mathrm{E}-03$ & $1.62 \mathrm{E}-15$ & $1.62 \mathrm{E}-15$ & $6.61 \mathrm{E}-03$ & $3.25 \mathrm{E}+00$ & $1.62 \mathrm{E}-15$ & $7.66 \mathrm{E}-02$ & $1.62 \mathrm{E}-15$ & $1.62 \mathrm{E}-15$ \\
\hline $\mathrm{YLD}_{\mathrm{LD}}$ & $3.65 \mathrm{E}+00$ & $3.75 \mathrm{E}-03$ & $1.09 \mathrm{E}-15$ & $1.09 \mathrm{E}-15$ & $3.75 \mathrm{E}-03$ & $3.65 \mathrm{E}+00$ & $1.09 \mathrm{E}-15$ & $1.14 \mathrm{E}-01$ & $1.09 \mathrm{E}-15$ & $1.09 \mathrm{E}-15$ \\
\hline $\mathrm{YLD}_{\mathrm{HD}}$ & $1.84 \mathrm{E}+00$ & $1.13 \mathrm{E}-04$ & $3.38 \mathrm{E}-16$ & $3.38 \mathrm{E}-16$ & $2.95 \mathrm{E}-03$ & $1.84 \mathrm{E}+00$ & $1.19 \mathrm{E}-03$ & $6.13 \mathrm{E}-02$ & $3.38 \mathrm{E}-16$ & $3.38 \mathrm{E}-16$ \\
\hline $\mathrm{YLD}_{\mathrm{ATR}}$ & $2.62 \mathrm{E}-17$ & $2.62 \mathrm{E}-17$ & $2.62 \mathrm{E}-17$ & $2.62 \mathrm{E}-17$ & $1.37 \mathrm{E}-01$ & $1.37 \mathrm{E}-01$ & $1.37 \mathrm{E}-01$ & $1.37 \mathrm{E}-01$ & $2.62 \mathrm{E}-17$ & $2.62 \mathrm{E}-17$ \\
\hline $\mathrm{SG}_{\mathrm{LPG}}$ & $1.29 \mathrm{E}-09$ & $1.29 \mathrm{E}-09$ & $1.29 \mathrm{E}-09$ & $1.29 \mathrm{E}-09$ & $5.32 \mathrm{E}-08$ & $5.32 \mathrm{E}-08$ & $5.32 \mathrm{E}-08$ & $5.32 \mathrm{E}-08$ & $1.29 \mathrm{E}-09$ & $1.29 \mathrm{E}-09$ \\
\hline $\mathrm{SG}_{\mathrm{N}}$ & $2.17 \mathrm{E}-05$ & $1.36 \mathrm{E}-07$ & $7.89 \mathrm{E}-08$ & 7.89E-08 & $1.36 \mathrm{E}-07$ & $2.17 \mathrm{E}-05$ & $7.89 \mathrm{E}-08$ & $5.94 \mathrm{E}-07$ & $7.89 \mathrm{E}-08$ & $7.89 \mathrm{E}-08$ \\
\hline $\mathrm{SG}_{\mathrm{K}}$ & $2.87 \mathrm{E}-05$ & $2.47 \mathrm{E}-07$ & $8.51 \mathrm{E}-08$ & $8.51 \mathrm{E}-08$ & $2.47 \mathrm{E}-07$ & $2.87 \mathrm{E}-05$ & $8.51 \mathrm{E}-08$ & $7.61 \mathrm{E}-07$ & $8.51 \mathrm{E}-08$ & $8.51 \mathrm{E}-08$ \\
\hline $\mathrm{SG}_{\mathrm{LD}}$ & $1.32 \mathrm{E}-05$ & 4.52E-08 & $1.37 \mathrm{E}-08$ & $1.37 \mathrm{E}-08$ & $4.52 \mathrm{E}-08$ & $1.32 \mathrm{E}-05$ & $1.37 \mathrm{E}-08$ & $3.22 \mathrm{E}-07$ & $1.37 \mathrm{E}-08$ & $1.37 \mathrm{E}-08$ \\
\hline $\mathrm{SG}_{\mathrm{HD}}$ & 7.94E-06 & $1.15 \mathrm{E}-08$ & $3.44 \mathrm{E}-09$ & $3.44 \mathrm{E}-09$ & $1.15 \mathrm{E}-08$ & 7.94E-06 & $3.44 \mathrm{E}-09$ & $2.47 \mathrm{E}-07$ & $3.44 \mathrm{E}-09$ & $3.44 \mathrm{E}-09$ \\
\hline $\mathrm{SG}_{\mathrm{ATR}}$ & $2.70 \mathrm{E}-09$ & $2.70 \mathrm{E}-09$ & $2.70 \mathrm{E}-09$ & $2.70 \mathrm{E}-09$ & $2.70 \mathrm{E}-09$ & $2.70 \mathrm{E}-09$ & $2.70 \mathrm{E}-09$ & $2.70 \mathrm{E}-09$ & $2.70 \mathrm{E}-09$ & $2.70 \mathrm{E}-09$ \\
\hline $\mathrm{S}_{\mathrm{N}}$ & $3.24 \mathrm{E}-06$ & $1.30 \mathrm{E}-07$ & $1.91 \mathrm{E}-08$ & $1.91 \mathrm{E}-08$ & $1.30 \mathrm{E}-07$ & $3.24 \mathrm{E}-06$ & $1.91 \mathrm{E}-08$ & $1.34 \mathrm{E}-07$ & $1.91 \mathrm{E}-08$ & $1.91 \mathrm{E}-08$ \\
\hline $\mathrm{S}_{\mathrm{K}}$ & $8.80 \mathrm{E}-05$ & $3.04 \mathrm{E}-07$ & $1.18 \mathrm{E}-07$ & $1.18 \mathrm{E}-07$ & $3.04 \mathrm{E}-07$ & $8.80 \mathrm{E}-05$ & $1.18 \mathrm{E}-07$ & $3.89 \mathrm{E}-06$ & $1.18 \mathrm{E}-07$ & $1.18 \mathrm{E}-07$ \\
\hline $\mathrm{S}_{\mathrm{LD}}$ & $5.21 \mathrm{E}-04$ & $1.61 \mathrm{E}-06$ & $7.40 \mathrm{E}-07$ & $7.40 \mathrm{E}-07$ & $1.61 \mathrm{E}-06$ & $5.21 \mathrm{E}-04$ & $7.40 \mathrm{E}-07$ & $1.98 \mathrm{E}-05$ & $7.40 \mathrm{E}-07$ & $7.40 \mathrm{E}-07$ \\
\hline $\mathrm{S}_{\mathrm{HD}}$ & $1.58 \mathrm{E}-04$ & $2.11 \mathrm{E}-06$ & $4.66 \mathrm{E}-07$ & $4.66 \mathrm{E}-07$ & $2.22 \mathrm{E}-06$ & $1.74 \mathrm{E}-04$ & $4.66 \mathrm{E}-07$ & $2.49 \mathrm{E}-05$ & $4.66 \mathrm{E}-07$ & 4.66E-07 \\
\hline $\mathrm{S}_{\mathrm{ATR}}$ & $1.23 \mathrm{E}-07$ & $1.23 \mathrm{E}-07$ & $1.23 \mathrm{E}-07$ & $1.23 \mathrm{E}-07$ & $3.15 \mathrm{E}-05$ & $3.15 \mathrm{E}-05$ & $3.15 \mathrm{E}-05$ & $3.15 \mathrm{E}-05$ & $1.23 \mathrm{E}-07$ & $1.23 \mathrm{E}-07$ \\
\hline
\end{tabular}


Table S10: Least Squares errors for Data Set 2 using the ISW model

\begin{tabular}{|c|c|c|c|c|c|c|c|c|c|c|}
\hline & Model 1 & Model 2 & Model 3 & Model 4 & Model 5 & Model 6 & Model 7 & Model 8 & Model 9 & $\begin{array}{c}\text { Model } \\
10\end{array}$ \\
\hline $\mathrm{YLD}_{\mathrm{LPG}}$ & $1.50 \mathrm{E}-29$ & $1.50 \mathrm{E}-29$ & $1.50 \mathrm{E}-29$ & $1.50 \mathrm{E}-29$ & $2.39 \mathrm{E}-29$ & $2.39 \mathrm{E}-29$ & $2.39 \mathrm{E}-29$ & $2.39 \mathrm{E}-29$ & $1.50 \mathrm{E}-29$ & $1.50 \mathrm{E}-29$ \\
\hline $\mathrm{YLD}_{\mathrm{N}}$ & $8.20 \mathrm{E}-01$ & $2.18 \mathrm{E}-03$ & $7.03 \mathrm{E}-16$ & $7.03 \mathrm{E}-16$ & $2.18 \mathrm{E}-03$ & $8.20 \mathrm{E}-01$ & $7.03 \mathrm{E}-16$ & $9.63 \mathrm{E}-03$ & $7.03 \mathrm{E}-16$ & $7.03 \mathrm{E}-16$ \\
\hline $\mathrm{YLD}_{\mathrm{K}}$ & $3.31 \mathrm{E}+00$ & $5.76 \mathrm{E}-03$ & $1.60 \mathrm{E}-15$ & $1.60 \mathrm{E}-15$ & $5.76 \mathrm{E}-03$ & $3.31 \mathrm{E}+00$ & $1.60 \mathrm{E}-15$ & $7.38 \mathrm{E}-02$ & $1.60 \mathrm{E}-15$ & $1.60 \mathrm{E}-15$ \\
\hline YLD $_{\mathrm{LD}}$ & $4.82 \mathrm{E}+00$ & $2.56 \mathrm{E}-03$ & $1.46 \mathrm{E}-15$ & $1.46 \mathrm{E}-15$ & $2.56 \mathrm{E}-03$ & $4.82 \mathrm{E}+00$ & $1.46 \mathrm{E}-15$ & $1.23 \mathrm{E}-01$ & $1.46 \mathrm{E}-15$ & $1.46 \mathrm{E}-15$ \\
\hline $\mathrm{YLD}_{\mathrm{HD}}$ & $1.92 \mathrm{E}+00$ & $1.44 \mathrm{E}-04$ & $4.04 \mathrm{E}-16$ & $4.04 \mathrm{E}-16$ & $3.14 \mathrm{E}-03$ & $1.92 \mathrm{E}+00$ & $1.88 \mathrm{E}-03$ & $7.59 \mathrm{E}-02$ & $4.04 \mathrm{E}-16$ & 4.04E-16 \\
\hline $\mathrm{YLD}_{\mathrm{ATR}}$ & $8.32 \mathrm{E}-16$ & $8.32 \mathrm{E}-16$ & $8.32 \mathrm{E}-16$ & $8.32 \mathrm{E}-16$ & $1.22 \mathrm{E}-01$ & $1.22 \mathrm{E}-01$ & $1.22 \mathrm{E}-01$ & $1.22 \mathrm{E}-01$ & $8.32 \mathrm{E}-16$ & $8.32 \mathrm{E}-16$ \\
\hline $\mathrm{SG}_{\mathrm{LPG}}$ & $1.73 \mathrm{E}-09$ & $1.73 \mathrm{E}-09$ & $1.73 \mathrm{E}-09$ & $1.73 \mathrm{E}-09$ & 4.47E-08 & 4.47E-08 & 4.47E-08 & 4.47E-08 & $1.73 \mathrm{E}-09$ & $1.73 \mathrm{E}-09$ \\
\hline $\mathrm{SG}_{\mathrm{N}}$ & $2.62 \mathrm{E}-05$ & $1.61 \mathrm{E}-07$ & $1.04 \mathrm{E}-07$ & $1.04 \mathrm{E}-07$ & $1.61 \mathrm{E}-07$ & $2.62 \mathrm{E}-05$ & $1.04 \mathrm{E}-07$ & $5.16 \mathrm{E}-07$ & $1.04 \mathrm{E}-07$ & $1.04 \mathrm{E}-07$ \\
\hline $\mathrm{SG}_{\mathrm{K}}$ & $3.85 \mathrm{E}-05$ & $1.67 \mathrm{E}-07$ & $1.48 \mathrm{E}-07$ & $1.48 \mathrm{E}-07$ & $1.67 \mathrm{E}-07$ & $3.85 \mathrm{E}-05$ & $1.48 \mathrm{E}-07$ & $8.09 \mathrm{E}-07$ & $1.48 \mathrm{E}-07$ & $1.48 \mathrm{E}-07$ \\
\hline $\mathrm{SG}_{\mathrm{LD}}$ & $1.27 \mathrm{E}-05$ & $5.11 \mathrm{E}-08$ & $1.73 \mathrm{E}-08$ & $1.73 \mathrm{E}-08$ & $5.11 \mathrm{E}-08$ & $1.27 \mathrm{E}-05$ & $1.73 \mathrm{E}-08$ & $4.10 \mathrm{E}-07$ & $1.73 \mathrm{E}-08$ & $1.73 \mathrm{E}-08$ \\
\hline $\mathrm{SG}_{\mathrm{HD}}$ & $8.40 \mathrm{E}-06$ & $9.54 \mathrm{E}-09$ & $3.99 \mathrm{E}-09$ & $3.99 \mathrm{E}-09$ & $9.54 \mathrm{E}-09$ & $8.40 \mathrm{E}-06$ & 3.99E-09 & $3.23 \mathrm{E}-07$ & 3.99E-09 & $3.99 \mathrm{E}-09$ \\
\hline $\mathrm{SG}_{\mathrm{ATR}}$ & $1.93 \mathrm{E}-09$ & $1.93 \mathrm{E}-09$ & $1.93 \mathrm{E}-09$ & $1.93 \mathrm{E}-09$ & $1.93 \mathrm{E}-09$ & $1.93 \mathrm{E}-09$ & $1.93 \mathrm{E}-09$ & $1.93 \mathrm{E}-09$ & $1.93 \mathrm{E}-09$ & $1.93 \mathrm{E}-09$ \\
\hline $\mathrm{S}_{\mathrm{N}}$ & $3.89 \mathrm{E}-06$ & $1.59 \mathrm{E}-07$ & $2.72 \mathrm{E}-08$ & $2.72 \mathrm{E}-08$ & $1.59 \mathrm{E}-07$ & $3.89 \mathrm{E}-06$ & $2.72 \mathrm{E}-08$ & $1.56 \mathrm{E}-07$ & $2.72 \mathrm{E}-08$ & $2.72 \mathrm{E}-08$ \\
\hline $\mathrm{S}_{\mathrm{K}}$ & $1.17 \mathrm{E}-04$ & $3.19 \mathrm{E}-07$ & $1.34 \mathrm{E}-07$ & $1.34 \mathrm{E}-07$ & $3.19 \mathrm{E}-07$ & $1.17 \mathrm{E}-04$ & $1.34 \mathrm{E}-07$ & $4.56 \mathrm{E}-06$ & $1.34 \mathrm{E}-07$ & $1.34 \mathrm{E}-07$ \\
\hline $\mathrm{S}_{\mathrm{LD}}$ & $4.83 \mathrm{E}-04$ & $9.96 \mathrm{E}-07$ & $5.90 \mathrm{E}-07$ & $5.90 \mathrm{E}-07$ & $9.96 \mathrm{E}-07$ & 4.83E-04 & $5.90 \mathrm{E}-07$ & $2.63 \mathrm{E}-05$ & $5.90 \mathrm{E}-07$ & $5.90 \mathrm{E}-07$ \\
\hline $\mathrm{S}_{\mathrm{HD}}$ & $1.68 \mathrm{E}-04$ & $3.87 \mathrm{E}-06$ & $5.11 \mathrm{E}-07$ & $5.11 \mathrm{E}-07$ & $3.62 \mathrm{E}-06$ & $1.63 \mathrm{E}-04$ & $5.11 \mathrm{E}-07$ & $1.86 \mathrm{E}-05$ & $5.11 \mathrm{E}-07$ & $5.11 \mathrm{E}-07$ \\
\hline $\mathrm{S}_{\mathrm{ATR}}$ & $1.13 \mathrm{E}-07$ & $1.13 \mathrm{E}-07$ & $1.13 \mathrm{E}-07$ & $1.13 \mathrm{E}-07$ & $2.76 \mathrm{E}-05$ & $2.76 \mathrm{E}-05$ & $2.76 \mathrm{E}-05$ & $2.76 \mathrm{E}-05$ & $1.13 \mathrm{E}-07$ & $1.13 \mathrm{E}-07$ \\
\hline
\end{tabular}


Appendix D - Cross plots for Surrogate Model 3 using the ISW method

Figure $\mathrm{S} 1$ presents the cross plots for the $\mathrm{Y}_{\text {estimated }}\left(\mathrm{y}\right.$ axis) versus the $\mathrm{Y}_{\text {real }}$ ( $\mathrm{x}$ axis) for the yield, specific gravity, and sulfur content of all dependent variables in Surrogate Model 3 using the ISW method. The black line represents the $y=x$ function. 

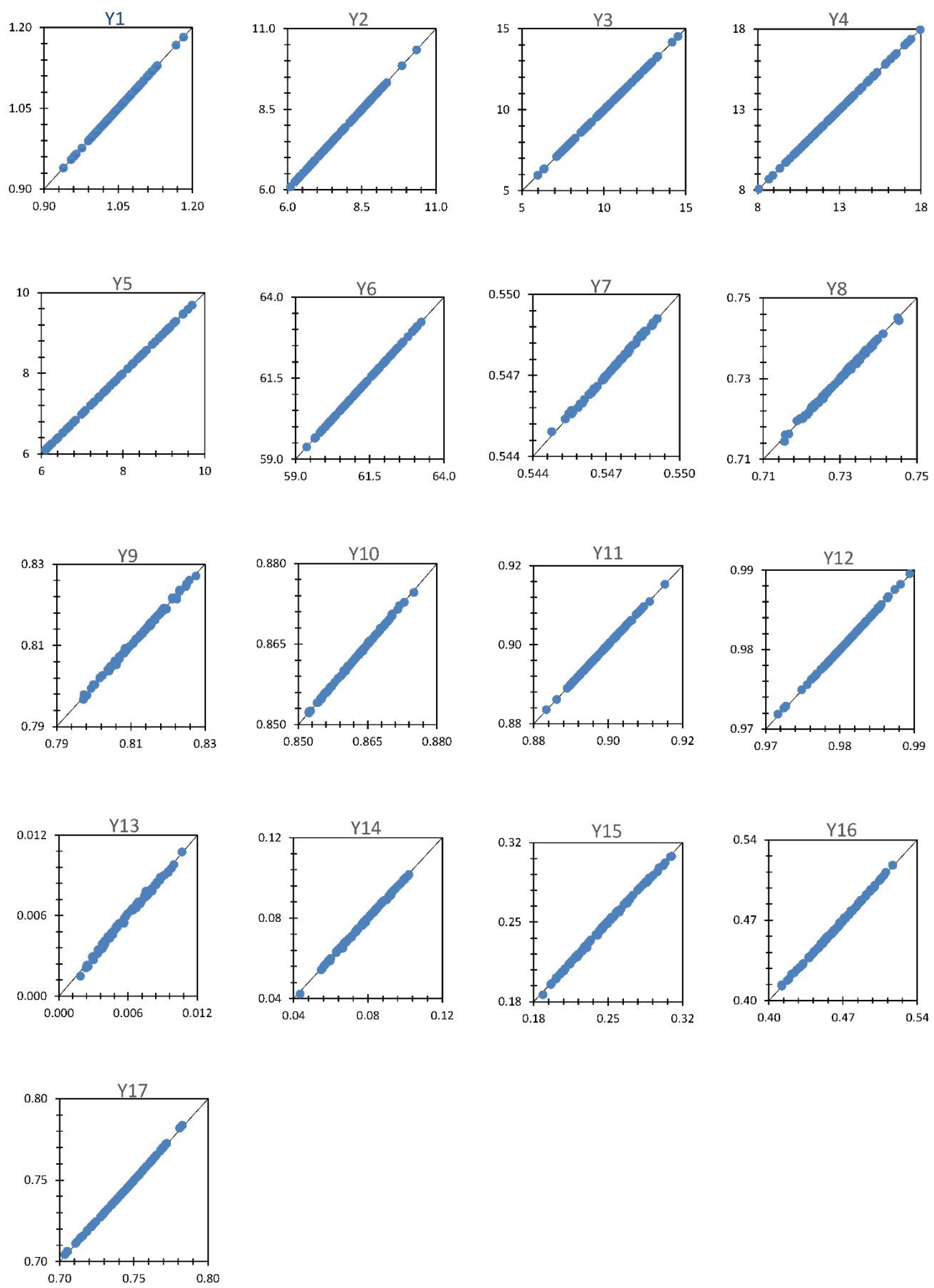

Figure S1: Plots for Surrogate Model 3 using the ISW method 


\section{Nomenclature}

\section{Continuous Variables}

$M P_{c u t}^{f c}$ : mass-based property of final cut

$M P_{c u t}^{i c}:$ mass-based property of intermediate cut

$M P I_{S w}$ : mass-based property of interface of swing-cut

$M P_{m c 1}$ : mass-based property of adjacent light micro cut

$M P_{m c 2}:$ mass-based property of adjacent heavy micro cut

$M P_{s w}:$ mass-based property of swing-cut (bulk)

$M P_{S W}^{\text {light }}$ : mass-based property of light fraction of swing-cut

$M P_{S w}^{\text {heavy }}$ : mass-based property of heavy fraction of swing-cut

$M P I_{S W}^{\text {light }}$ : mass-based property of interface of light fraction of swing-cut

$M P I_{S w}^{\text {heavy }}$ : mass-based property of interface of heavy fraction of swing-cut

$Q_{c, c d u}$ : inlet volumetric feed flow for crude c incoming to cdu

$Q_{c d u}$ : overall volumetric feed flow for cdu

$Q_{c u t}^{f c}$ : volumetric flow of final distillate $c u t$

$Q_{m c}$ : volumetric flow of micro cut mc (pseudo-components)

$Q_{c u t}^{i c}$ : volumetric flow of intermediate distillate $c u t$

$Q_{s w 1}$ to $Q_{s w 3}$ : volumetric flow of swing-cuts (bulk)

$Q_{s w 1}^{\text {light }}$ to $Q_{s w 3}^{\text {light. }}$ : volumetric flow of light fraction of swing-cuts

$Q_{s w 1}^{\text {heavy }}$ to $Q_{s w 3}^{\text {heavy: }}$ volumetric flow of heavy fraction of swing-cuts

$Q_{s w}$ : volumetric flow of swing-cut

$Q_{m c 1}$ : volumetric flow of adjacent light micro cut

$Q_{m c 2}$ : volumetric flow of adjacent heavy micro cut

$Q_{S W}^{\text {light }}$ : volumetric flow of light fraction of swing-cut

$Q_{s w}^{h e a v y}$ : volumetric flow of heavy fraction of swing-cut

$V P_{c u t}^{f c}$ : volume-based property of final cut 
$V P_{c u t}^{i c}$ : volume-based property of intermediate cut

$V P I_{s w}$ : volume-based property of interface of swing-cut

$V P_{m c 1}$ : volume-based property of adjacent light micro cut

$V P_{m c 2}$ : volume-based property of adjacent heavy micro cut

$V P_{s w}$ : volume-based property of swing-cut (bulk)

$V P_{s W}^{\text {light }}$ : volume-based property of light fraction of swing-cut

$V P_{s w}^{h e a v y}$ : volume-based property of heavy fraction of swing-cut

$V P I_{S W}^{\text {light }}$ : volume-based property of interface of light fraction of swing-cut

$V P I_{S w}^{\text {heavy: }}$ volume-based property of interface of heavy fraction of swing-cut

$Y L D_{m c}:$ yields of micro cut mc

\section{Sets}

$F C$ : final cuts of distillates

$I C$ : intermediate cuts of distillates

$M C_{f c}$ : micro cuts related to final cuts of distillates

$M C_{i c}$ : micro cuts related to intermediate cuts of distillates

SW: swing-cuts

\section{Subscripts}

ATR: atmospheric residue

$c$ : crude oil

$C D U$ : crude distillation unit

cut: final distillate cut

$H D$ : heavy diesel

heavy: heavy fraction of swing-cut

$K$ : kerosene

$L D$ : light diesel

light: light fraction of swing-cut

$m c$ : micro cut 
$N$ : naphtha

$s w$ : swing-cut

sw1: swing-cut 1, between naphtha and kerosene

sw2: swing-cut 2, between kerosene and light diesel

sw3: swing-cut 3, between light diesel and heavy diesel

$L P G$ : liquefied petroleum gas

\section{Superscripts}

$f c$ : final cut

heavy: heavy fraction of swing-cut

$i c$ : intermediate cut

light: light fraction of swing-cut

\section{Abbreviations}

ATR: atmospheric residue

CDU: crude distillation unit

CSW: conventional swing-cut

FG: fuel gas

FY: fixed yields

HD: heavy diesel

ISW: improved swing-cut

$\mathrm{K}$ : kerosene

LD: light diesel

LPG: liquefied petroleum gas

$\mathrm{N}$ : naphtha

S: sulfur content

SG: specific gravity

SW1 to SW3: swing-cuts

$x_{1}$ to $x_{4}$ : independent variables for the four crude oils in the surrogate model

$x_{5}$ to $x_{7}$ : independent variables for the three swing-cuts in the surrogate model 
Y: dependent variable in the surrogate model YLD: yield 